\title{
The in-Human Host and in-Mosquito Dynamics of Malaria Parasites With Immune Responses
}

\author{
Mohamed A. Selemani ${ }^{1,3}$, Livingstone S. Luboobi ${ }^{1,2}$ and Yaw Nkansah-Gyekye ${ }^{1}$ \\ ${ }^{1}$ Department of Mathematics, Nelson Mandela African Institution of Science and Technology (NM-AIST), Arusha, Tanzania \\ ${ }^{2}$ Department of Mathematics, Makerere University, P.O. Box 7062, Kampala, Uganda \\ ${ }^{3}$ Department of Mathematics and Statistics, Sokoine University of Agriculture, Morogoro, Tanzania
}

Received: 16 December 2016, Accepted: 13 May 2017

Published online: 25 August 2017.

\begin{abstract}
In this study, a mathematical model for the in-human host and in-mosquito dynamics of malaria parasite with immune responses was formualeted and analyzed. A positive invariant region of the model was established, and a basic reproduction number $\mathscr{R}_{0}$, of the model was computed. Existence and stability of two non-negative equilibrium points: malaria free equilibrium (MFE) and malaria infection equlibrium (MIE) were established. We, also proved that MFE is locally asymptotically stable if $\mathscr{R}_{0}<1$ and globally asymptotically stable (GAS) if $\mathscr{R}_{0} \leq 1$. Numerical simulations prove that MIE exists and is GAS. Moreover, our results revealed that immunity has significant influence on lowering malaria infection at blood and mosquito stages. However, an insignificant effect of immunity on both cells and parasites at liver stage infection was observed. Furthermore, the model depicts that infection decreases as lifespan of immune cells increases. The impact of immune cells to suppress production of merozoites is noted to be higher than that of antibodies to block invasion of sporozoites and merozoites.
\end{abstract}

Keywords: Malaria parasites, in-human host and in-mosquito dynamics, immune responses.

\section{Introduction}

In human, malaria infection is initiated by a bite from a parasite-carrying mosquito, where sporozoites are injected into bloodstream and quickly migrate to the liver, where they penetrate hepatic liver cells (HLCs) and develop into schizonts that give rise to thousands of merozoites into bloodstream [7]. Merozoites invade red blood cells (RBCs), that develop into erythrocytic schizonts which finally burst and release an average of 16 merozoites [20] which either re-invade new RBCs or switch to sexual form termed gametocytes. Human-mosquito infection of malaria begins through ingestion of gametocytes into parasite-free mosquito during its blood-meal. Ingested gametocytes then fuse and develop into oocysts, where each oocyst ruptures and releases an average of 1000 sporozoites [18] that are responsible for new mosquito-human infection.

A major role of the human immune system is to defend a body against the infection-causing organisms, called pathogens. Human immune system has two main components: innate immunity and adaptive immunity. Innate immunity defends the body against any pathogenic invasion, while adaptive immunity provides protection against a specific pathogen, and usually comes into action after the infections outrun the innate immunity. Innate immune responses include macrophages, interferon and natural killer (NK) cells, while T-lymphocyte (cytotoxic $\mathrm{T}$ and helper T) and B-lymphocyte (B-cells) are some elements of adaptive immune responses. Cellular-mediated responses involves cell effectors such as cytotoxic $\mathrm{T}\left(\mathrm{CD} 8^{+} \mathrm{T}\right)$ and NK cells to kill intracellular pathogens while humoral responses involve effector molecules such as antibodies (secreted by B-cells) to clear free pathogens in body's fluid such blood. 
In malaria infection, both innate and adaptive immune responses are stimulated $[16,15]$ to obstruct parasites by either preventing the re-invasion of parasite or increasing the death rate of infected cells $[12,16]$, and both humoral and cell-mediated immune effector mechanisms are involved in immunology of malaria [15,14]. Antibodies neutralize the sporozoites and merozoites and inhibit sporozoites' invasion to HLCs [14,15] and merozoites' invasion to RBCs [20,14]. They also restrain the parasite growth [6,14]. Macrophages are activated by NK cells to intensify phagocytosis and clearance of intra-erythrocytic parasites [3]. The IFN- $\gamma$ produced by $\mathrm{CD} 8^{+}$T cells (in help of $\mathrm{CD} 4^{+}$) inhibit growth of, and kill intrahepatic parasites [14,3,15]. Moreover, antibodies and complement system that are ingested by mosquito during blood meal mediate the lysis of gametocytes and inhibit development of parasite in the mosquito [15].

In recent years, there has been increasing interest in mathematical models on intra host dynamics of malaria with immune responses $[16,20,6]$ and references therein. Some of these studies ignored the absorption effect of merozoites into RBC [16]. However, some authors incorporate this effect since during malaria infection parasite penetrates into healthy cells. Therefore, in this case both populations (parasites and uninfected cells) decrease $[2,20,6]$. Moreover, the clearance of free parasites or infected cells by immune responses has been modelled either as simple mass-action [20,6] which is unbounded function or using the Michaelis-Menten-Monod function (MMMF) which is nonlinear-bounded [16]. Furthermore, [6] incorporate the effects of antibodies to inhibit parasite's growth or block invasion of host's cells by parasites using MMMF.

However, none of these studies discussed the liver stage dynamics of malaria parasites. As it has been stated in previous paragraph, some studies did not incoporate the absorption effect of parasites into uninfected cells. Moreover, some of these models ignored either the saturation effect on cell proliferation and/or suppression of parasites replication. In this study, we formulate a mathematical model for the in-human host and in mosquito dynamics of malaria with immune responses, where the MMMF is used to describe the effect of both immune responses on clearance of infected cells and free parasites. The effect of antibodies on supressing the replication of parasites at liver and blood stages of malaria infection is included. Lastly, we incoroprate the effect of antibodies picked-up by mosquito in mediating the lysis of gametocytes and preventing parasite's development in the mosquito.

\section{Model formulation}

\subsection{Model desription}

In development of this model, we extend the model by [19] by incorporating the effect of immune system. The dynamics of interactions of malaria parasites in-mosquito vector and in-human host with immune responses are described using a system of nonlinear ordinary differential equations. Variables involved in this model are: the uninfected hepatocytic liver cells (uHLCs), $H$; infected hepatocytic liver cells (iHLCs), $I_{h}$; hepatic schizonts, $T_{h}$; merozoites, $M$; uninfected red blood cells (uRBCs), $R$; infected red blood cells (iRBCs), $I_{r}$; erythrocytic schizonts, $T_{r}$; gametocytes, $G_{b}$; gametes, $G_{m}$; and oocysts, $C$. Others are sporozoites in mosquito's salivary gland, $S_{m}$; sporozoites in human, $S_{h}$; Immune cells (which includes IFN- $\gamma, \mathrm{CD} 8^{+}$and $\mathrm{CD} 4^{+}$T, B and NK cells, macrophages etc) against liver stage and blood stage infections, $Z_{1}$ and $Z_{2}$ respectively; and antibodies, $B$.

Sporozoites, $S_{h}$ are injected into uninfected human host at a constant rate $a b v$, during a blood meal of infected mosquito, where $a$ is number of mosquito bites per individual, $b$ is number of sporozoites injected per bite and $v$ is probability that a mosquito bite is infective to human. Sporozoites attack the uHLCs at the rate $\beta_{1} S_{h} H /\left(1+k_{1} B\right)$, where $\beta_{1}$ is sporozoites' infection rate to uHLCs and $k_{1}$ is efficiency of antibodies to block invasion of uHLCs. 
The uHLCs are constantly recruited at rate $\Lambda_{h}$, die naturally at rate $\mu_{h} H$ and reduced at rate $\beta_{1} S_{h} H /\left(1+k_{1} B\right)$ due to infection by sporozoites, and they are killed by immune cells (macrophages) at a rate $\sigma_{s h} Z_{1} S_{h} /\left(1+\pi_{s h} S_{h}\right)$, where $\sigma_{s h}$ is rate of successful removal of intra-human sporozoites by immune cells and $1 / \pi_{s h}$ is a half saturation constant of sporozoites. The iHLCs increase at a rate $\beta_{1} S_{h} H /\left(1+k_{1} B\right)$ due to infection of uHLCs and die at a rate $\mu_{i h} I_{h}$. They progress to schizonts at a rate $\alpha_{1} I_{h}$ and killed by immune cells (IFN- $\gamma$, CD8 ${ }^{+}$, NK) at a rate $\sigma_{i h} Z_{1} I_{h} /\left(1+\pi_{i h} I_{h}\right)$, where $\sigma_{i h}$ is rate of successful removal of iHLCs by immune cells and $1 / \pi_{i h}$ is a half saturation constant of $I_{h}$. The hepatic schizonts die naturally at a rate $\mu_{t h} T_{h}$, rupture to release merozoites a rate $\delta_{1} T_{h}$, and cleared by immune cells (IFN- $\gamma$, $\left.\mathrm{CD}^{+}, \mathrm{NK}\right)$ at a rate $\sigma_{t h} Z_{1} T_{h} /\left(1+\pi_{t h} T_{h}\right)$, where $\sigma_{t h}$ is rate of successful removal of hepatic schizonts by immune cells and $1 / \pi_{t h}$ is a half saturation constant of hepatic schizonts.

Merozoites are released from the hepatic schizonts at a rate $r_{1} \delta_{1} T_{h} /\left(1+c_{1} Z_{1}\right)$, where $c_{1}$ is efficiency of immune cells (IFN- $\gamma$ and $\mathrm{CD}^{+}$) to inhibit the merozoites' production. They invade uRBCs at a rate $\beta_{2} R M /\left(1+k_{2} B\right)$ and they die naturally at $\mu_{m} M$. The parameter $\beta_{2}$ is merozoites' infection rate to uRBCs and $k_{2}$ is efficiency of antibodies to inhibit the infection of uRBCs by merozoites. They are cleared by immune cells (macrophages activated by IFN- $\gamma$ ) at a rate $\sigma_{m} Z_{2} M /\left(1+\pi_{m} M\right)$, where $\sigma_{m}$ is rate of successful removal of merozoites by immune cells and $1 / \pi_{m}$ is a half saturation constant of merozoites.

The uRBCs are constantly recruited at a rate $\Lambda_{r}$ from the bone marrow. Their density is reduced by natural death at a rate $\mu_{r} R$ and due to the infection by merozoites at a rate $\beta_{2} R M /\left(1+k_{2} B\right)$. The iRBCs increases at a rate $\beta_{2} R M /\left(1+k_{2} B\right)$ and decreases due to death at a rate $\mu_{i r} I_{r}$ and due to progression to erythrocytic schizonts at a rate $\alpha_{2} T_{r}$. The immune cells (macrophages activated by IFN- $\gamma$ ) phagocytize the iRBCs at a rate $\sigma_{i r} Z_{2} I_{r} /\left(1+\pi_{i r} I_{r}\right)$, where $\sigma_{i r}$ is rate of successful removal of iRBCs by immune cells and $1 / \pi_{i r}$ is a half saturation constant of iRBCs. The erythrocytic schizonts die at rate $\mu_{t r} T_{r}$ and rupture to release new merozoites at rate $\delta_{2} T_{r}$, which they initiate a series of repetitive cycles to infect other uRBCs. Proportion $p$, of these newly released merozoites procceds with asexual replication cycle at a rate $\operatorname{pr}_{2} \delta_{2} T_{r} /\left(1+c_{2} Z_{2}\right)$, while the other proportion $1-p$ switch to sexual form of parasites called gametocytes at a rate $(1-p) r_{2} \delta_{2} T_{r} /\left(1+c_{2} Z_{2}\right)$. The parameter $c_{2}$ is efficiency of immune cells to inhibit the production of intra-erythrocytic merozoites or gametocytes. They are also killed by immune cells (macrophages) at a rate $\sigma_{t r} Z_{2} I_{r} /\left(1+\pi_{t r} T_{r}\right)$. The parameter $\sigma_{t r}$ is rate of successful removal of erythrocytic schizonts by immune cells and $1 / \pi_{t r}$ is a half saturation constant of erythrocytic schizonts.

The gametocytes in blood stream increases at a rate $(1-p) r_{2} \delta_{2} T_{r} /\left(1+c_{2} Z_{2}\right)$ and decrease by natural death at a rate $\mu_{g b} G_{b}$, for being ingested by mosquito at a rate $q \omega G_{b}$ and being cleared by immune cells at a rate $\sigma_{g b} Z_{2} G_{b} /\left(1+\pi_{g b} G_{b}\right)$. The parameters $\sigma_{g b}$ and $1 / \pi_{g b}$ are respectively, the rate of successful removal of gametocytes by immune cells and the half saturation constant of gametocytes. The gametes in mosquitoes are recruited at a rate $\rho q \omega G_{b} /\left(1+k_{3} B\right)$, where $\rho$ is number of bites a mosquito can make during its lifetime and $k_{3}$ is efficiency of antibodies picked up by mosquito during its blood meal to mediate lysis of gametocytes and prevent parasite's development in mosquito [15]. Gametes decrease by natural death at rate $\mu_{g m} G_{m}$ and progression to oocysts at a rate, $\alpha_{3} G_{m}$. The oocysts rupture to release an avarage of $r_{3}$ sporozoites per oocyst at a rate, $\delta_{3} C$ and die at a rate $\mu_{c} C$. The released sporozoites, $S_{m}$ migrate to salivary glands where they either naturally die at a rate $\mu_{s m} S_{m}$ or injected into a new host at rate $a b v$. Table 1 below summarizes the variables of the model and their biological descriptions. 
Table 1: Variables and their descriptions

\begin{aligned} & \hline Variable Description \\ & \hline \hline$S_{h}:$ number of sporozoites injected to the liver \\ &$H:$ number of uninfected HLCs \\ &$I_{h}:$ number of infected HLCs \\ &$T_{h}:$ number of schizonts developed from infected HLCs \\ &$T_{r}:$ number of schizonts developed from infected RBCs \\ &$M:$ number of merozoites in bloodstream \\ &$R:$ number of uninfected RBCs \\ &$I_{r}:$ number of infected RBCs \\ &$G_{b}:$ number of gametocytes in the bloodstream \\ &$G_{m}:$ number of gametes in the mosquito \\ &$C:$ number of oocysts \\ &$S_{m}:$ number of sporozoites in mosqouito's salivary gland \\ &$Z_{1}:$ number of immune cells that fight against malaria infection at the liver stage \\ &$Z_{2}:$ number of immune cells that fight against malaria infection at the blood stage \\ &$B:$ number of antibodies \\ & \hline\end{aligned}

\subsection{Model Assumptions}

In development of this model, we assume the following

(i) Both HLCs and RBCs are constantly recruited from bone marrow and they die naturally.

(ii) Infections of HLCs and RBCs are reduced by presence of immune responses.

(iii) Number of gametocytes ingested by mosquito depends on gametocytes' density in bloodstream, while number of sporozoites injected by mosquito is independent of sporozoites' density in salivary glands [8].

(iv) Death rates of infected cells are higher than that of uninfected ones.

(v) The injected sporozoites and the released merozoites do either die or successfully infect HCLs and RBCs respectively.

(vi) The ingested gametocytes either die or macro- and micro- gametes successfully fuse.

(vii) Constant proportion of asexual parasites converts to gametocytes within each cycle.

(viii) The cycle starts when the infected mosquito bites the human.

(ix) Bite of an infected mosquito onto an infected host is neglected.

(x) Survival of mosquito depends on human blood for developing their eggs.

(xi) For simplicity, all immune cells that fight against malaria infection in human are grouped into two compartments: immune cells against liver-stage infection and immune cells against blood-stage infection. All malaria-specific antibodies are considered as single compartment.

(xii) Production of immune cells is due constant recruitement from hematopoietic stem cell in the bone marrow, and stimulation by presence of sprozoites, schizonts, infected HLCs and RBCs, merozoites and gametocytes. They natural die at constant rate.

(xiii) Proliferation of antibodies that inhibit invasion of HLCs and RBCs depends only presence of sporozoites and merozoites. They die constantly.

(xiv) Antibodies ingested with gametocytes during the blood meal prevent the fusion of gametocytes (macro- and microgametes) and other developmental stages of parasites within mosquito. 
Table 2: Model parameters and their description

\begin{tabular}{|c|c|c|c|}
\hline Par & Description & Value & References \\
\hline$a:$ & probability that a bite infects human & 0.75 & {$[19]$} \\
\hline$b:$ & number of mosquito bites per individual & 15 day $^{-1}$ & [19] \\
\hline$v:$ & number of sporozoites injected per bite & $10-20$ & [19] \\
\hline$\beta_{1}:$ & infection rate of HLCs by sporozoites & $0.001 \mu l c^{-1} d^{-1}$ & [19] \\
\hline$r_{1}:$ & number of merozoites per liver schizont & 10000 & [19] \\
\hline \multirow{2}{*}{$\alpha_{1}:$} & progression rate of infected HCLs & & \\
\hline & to schizonts & $0.125 d^{d a y^{-1}}$ & [19] \\
\hline$\delta_{1}:$ & rupture rate of liver schizonts & $0.0975 d a y^{-1}$ & [19] \\
\hline$\Lambda_{h}:$ & the recruitmet rate of HLCs & $3000 c \mu l^{-1} d^{-1}$ & [19] \\
\hline$\mu_{h}:$ & natural death rate of uninfected HLCs & $0.94 d a y^{-1}$ & [19] \\
\hline$\mu_{i h}:$ & death rate of infected HLCs & $0.95 d_{a y}{ }^{-1}$ & [19] \\
\hline$\mu_{t h}:$ & death rate of liver-schizonts & 0.029 day $^{-1}$ & [19] \\
\hline$\beta_{2}$ : & infection rate of RBCs by merozoites & $2 \times 10^{-6} \mu l c^{-1} d^{-1}$ & [19] \\
\hline$\delta_{2}:$ & rupture rate of blood schizonts & $0.115 d^{-1}$ & [19] \\
\hline \multirow[t]{2}{*}{$\alpha_{2}:$} & progression rate of infected $\mathrm{RBCs}$ & & \\
\hline & to schizonts & $0.145 d^{-1}$ & [19] \\
\hline \multirow{3}{*}{$\begin{array}{r}r_{2}: \\
q:\end{array}$} & number of merozoites per blood schizont & 16 & [10] \\
\hline & probability that a bite is infectious & & \\
\hline & to mosquito & 0.09 & [1] \\
\hline \multirow{3}{*}{$\begin{array}{c}\omega: \\
\rho:\end{array}$} & number of gametocytes ingested per bite & 10 & [19] \\
\hline & number of bites made by mosquito & & \\
\hline & in its lifetime & 3 & [19] \\
\hline$\Lambda_{r}:$ & the recruitmet rate of RBCs & $41500 c \mu l^{-1} d^{-1}$ & [16] \\
\hline$\mu_{r}:$ & death rate of uninfected RBCs & $0.02 d^{-1}$ & [10] \\
\hline$\mu_{i r}:$ & total death rate of uninfected $\mathrm{RBCs}$ & $0.025 d^{-1}$ & [9] \\
\hline$\mu_{t r}:$ & death rate of blood-schizonts & 0.185 & [19] \\
\hline$\mu_{m}:$ & death rate of merozoites & $48 d^{-1}$ & [16] \\
\hline$\mu_{g b}:$ & death rate of gametocytes & $6.25 \times 10^{-5} d^{-1}$ & [19] \\
\hline$\delta_{3}:$ & rupture rate of Oocysts & $0.05 d_{a y} y^{-1}$ & [19] \\
\hline$r_{3}:$ & number of sporozoites per Oocyst & 1000 & [19] \\
\hline$\alpha_{3}:$ & progresion rate of gametes to Oocysts & $0.07 d^{-1}$ & [19] \\
\hline$\mu_{g m}:$ & death rate of gametes & $0.052 d^{-1}$ & [19] \\
\hline$\mu_{c}:$ & death rate of Oocysts & $0.024 d^{-1}$ & [19] \\
\hline$\mu_{s m}:$ & death rate of sporozoites in mosqouito & $40 d^{-1}$ & [19] \\
\hline \multirow{3}{*}{$\begin{array}{r}\mu_{s h}: \\
p:\end{array}$} & death rate of sporozoites in human liver & $1.2 \times 10^{-11} d^{-1}$ & [19] \\
\hline & proportion of asexual that differentiate & & \\
\hline & to merozoites & 0.926 & [19] \\
\hline$\Lambda_{z_{1}}:$ & the recruitmet rate of immune cells in the liver & $30 c \mu l^{-1} d^{-1}$ & Estimated \\
\hline$\Lambda_{z_{2}}:$ & the recruitmet rate of immune cells in the blood & $30 c \mu l^{-1} d^{-1}$ & [6] \\
\hline$\mu_{z_{1}}:$ & death rate of immune cells & 1.5 & Estimated \\
\hline$\mu_{z_{2}}:$ & death rate of immune cells & 1.53 & [6] \\
\hline$\mu_{b}:$ & deterioration rate of antibodies & 0.4 & [6] \\
\hline$c_{1}:$ & efficiency of immune cells to suppress & & \\
\hline
\end{tabular}


Table 2 - Continued from previous page

\begin{tabular}{|c|c|c|c|}
\hline Par & Description & Value & References \\
\hline & the production of $M$ from liver-schizonts & $10^{-5}$ & Estimated \\
\hline$c_{2}:$ & efficiency of immune cells to suppress & & \\
\hline & the production of $M$ from blood-schizonts & $3 \times 10^{-5}$ & Estimated \\
\hline$k_{1}:$ & efficiency of immune cells to inhibit invasion & & \\
\hline & of HLCs by sporozoites & 0.035 & Estimated \\
\hline$k_{2}:$ & $\begin{array}{l}\text { efficiency of immune cells to inhibit invasion } \\
\text { of RBCs by merozoites }\end{array}$ & 0.0015 & Estimated \\
\hline$k_{3}:$ & efficiency of immune cells to mediate & & \\
\hline$\sigma_{s h}:$ & $\begin{array}{l}\text { lysis of gametocytes and inhibit fertilization } \\
\text { rate at which sporozoites are cleared }\end{array}$ & 0.03 & Estimated \\
\hline & by immune cells & $9 \times 10^{-9}$ & Estimated \\
\hline$\sigma_{i h}:$ & rate at which infected HLCs are cleared & & \\
\hline & by immune cells & $9 \times 10^{-9}$ & Estimated \\
\hline$\sigma_{t h}:$ & $\begin{array}{l}\text { rate at which liver schizonts are cleared } \\
\text { by immune cells }\end{array}$ & $1 \times 10^{-8}$ & Estimated \\
\hline$\sigma_{m}:$ & $\begin{array}{l}\text { rate at which merozoites are cleared } \\
\text { by immune cells }\end{array}$ & $1 \times 10^{-8}$ & [16] \\
\hline$\sigma_{i r}:$ & $\begin{array}{l}\text { rate at which infected RBCs are cleared } \\
\text { by immune cells }\end{array}$ & $1 \times 10^{-8}$ & [16] \\
\hline$\sigma_{t r}:$ & $\begin{array}{l}\text { rate at which blood schizonts are cleared } \\
\text { by immune cells }\end{array}$ & $1 \times 10^{-8}$ & Estimated \\
\hline$\sigma_{g b}:$ & $\begin{array}{l}\text { rate at which gametocytes are cleared } \\
\text { by immune cells }\end{array}$ & $1 \times 10^{-8}$ & [4] \\
\hline$\varepsilon_{s h}:$ & $\begin{array}{l}\text { proliferation rate of immune cells } \\
\text { due to contact with sporozoites }\end{array}$ & $5 \times 10^{-5}$ & Estimated \\
\hline$\varepsilon_{i h}:$ & $\begin{array}{l}\text { proliferation rate of immune cells } \\
\text { due to contact with infected HLCs }\end{array}$ & $4.6 \times 10^{-5}$ & Estimated \\
\hline$\varepsilon_{t h}:$ & $\begin{array}{l}\text { proliferation rate of immune cells } \\
\text { due to contact with liver schizonts }\end{array}$ & $4.63 \times 10^{-5}$ & Estimated \\
\hline$\varepsilon_{m}:$ & $\begin{array}{l}\text { proliferation rate of immune cells } \\
\text { due to contact with merozoites }\end{array}$ & $4.69 \times 10^{-5}$ & [16] \\
\hline$\varepsilon_{i r}:$ & $\begin{array}{l}\text { proliferation rate of immune cells } \\
\text { due to contact with infected RBCs }\end{array}$ & $2.5 \times 10^{-5}$ & [16] \\
\hline$\varepsilon_{t r}:$ & $\begin{array}{l}\text { proliferation rate of immune cells } \\
\text { due to contact with blood schizonts }\end{array}$ & $2.5 \times 10^{-5}$ & Estimated \\
\hline$\varepsilon_{g b}:$ & $\begin{array}{l}\text { proliferation rate of immune cells } \\
\text { due to contact with gametocytes }\end{array}$ & $2.5 \times 10^{-5}$ & Estimated \\
\hline$\pi_{s h}:$ & $1 / \pi_{s h}$ saturation constant of sporozoites & $5 \times 10^{-4}$ & Estimated \\
\hline$\pi_{i h}:$ & $1 / \pi_{i h}$ saturation constant of infected HLCs & $5 \times 10^{-4}$ & Estimated \\
\hline$\pi_{t h}:$ & $1 / \pi_{t h}$ saturation constant of liver schizonts & $5 \times 10^{-4}$ & Estimated \\
\hline$\pi_{m}:$ & $1 / \pi_{m}$ saturation constant of merozoites & $7 \times 10^{-4}$ & [16] \\
\hline$\pi_{i r}:$ & $1 / \pi_{i r}$ saturation constant of infected RBCs & $5 \times 10^{-4}$ & [16] \\
\hline $\begin{aligned} \pi_{t r} & : \\
\eta_{1} & :\end{aligned}$ & $\begin{array}{l}1 / \pi_{t r} \text { saturation constant of blood schizonts } \\
\text { maximum rate of increase of antibodies }\end{array}$ & $5 \times 10^{-4}$ & Estimated \\
\hline
\end{tabular}


Table 2 - Continued from previous page

\begin{tabular}{clll}
\hline Par & Description & Value & References \\
\hline$\eta_{2}:$ & $\begin{array}{l}\text { due to presence of sporozoites } \\
\text { maximum rate of increase of antibodies } \\
\text { due to presence of merozoites }\end{array}$ & $1 \times 10^{-4}$ & Estimated \\
\end{tabular}

\subsection{Compartmental diagram}

Based on the dynamics described in Section 2.1 and the assumptions described in Section 2.2, the proposed model for the in-human host and in-mosquito dynamics of entire life cycle of malaria parasites with immune responses is shown in Figure 1, in which the variables and parameters are described in Table 1 and Table 2, respectively.

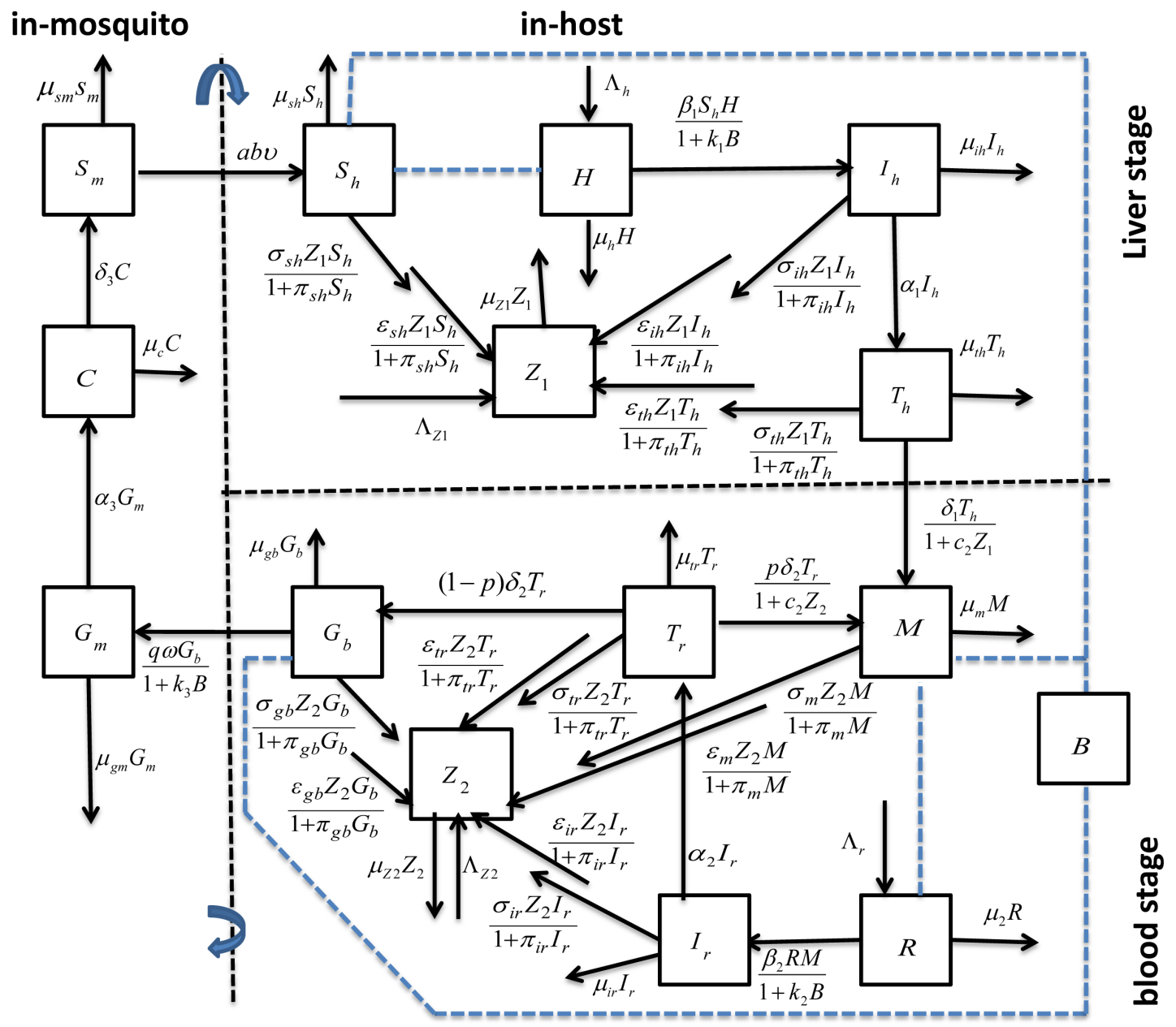

bite

Fig. 1: Compartmental model diagram for the in-human host and in-mosquito dynamics of malaria parasites with immune responses 


\subsection{Model Equations}

Based on the compartmental diagram illustrated in Figure 1 above, the in-human host and in-mosquito dynamics for the entire life cycle of malaria parasite with immune responses are governed by the following system of ordinary differential equations.

$$
\begin{aligned}
& \frac{\mathrm{d} H}{d t}=\Lambda_{h}-\frac{\beta_{1} S_{h} H}{1+k_{1} B}-\mu_{h} H \\
& \frac{\mathrm{d} I_{h}}{d t}=\frac{\beta_{1} S_{h} H}{1+k_{1} B}-\left(\alpha_{1}+\mu_{i h}\right) I_{h}-\frac{\sigma_{i h} Z_{1} I_{h}}{1+\pi_{i h} I_{h}} \\
& \frac{\mathrm{d} T_{h}}{d t}=\alpha_{1} I_{h}-\left(\delta_{1}+\mu_{t h}\right) T_{h}-\frac{\sigma_{t h} Z_{1} T_{h}}{1+\pi_{t h} T_{h}} \\
& \frac{\mathrm{d} M}{d t}=\frac{r_{1} \delta_{1} T_{h}}{1+c_{1} Z_{1}}+\frac{p r_{2} \delta_{2} T_{r}}{1+c_{2} Z_{2}}-\frac{\beta_{2} R M}{1+k_{2} B}-\frac{\sigma_{m} Z_{2} M}{1+\pi_{m} M}-\mu_{m} M \\
& \frac{\mathrm{d} R}{d t}=\Lambda_{r}-\frac{\beta_{2} R M}{1+k_{2} B}-\mu_{r} R \\
& \frac{\mathrm{d} I_{r}}{d t}=\frac{\beta_{2} R M}{1+k_{2} B}-\left(\alpha_{2}+\mu_{i r}\right) I_{r}-\frac{\sigma_{i r} Z_{2} I_{r}}{1+\pi_{i r} I_{r}} \\
& \frac{\mathrm{d} T_{r}}{d t}=\alpha_{2} I_{r}-\left(\delta_{2}+\mu_{t r}\right) T_{r}-\frac{\sigma_{t r} Z_{2} T_{r}}{1+\pi_{t r} T_{r}} \\
& \frac{\mathrm{d} G_{b}}{d t}=(1-p) \frac{r_{2} \delta_{2} T_{r}}{1+c_{2} Z_{2}}-\left(q \omega+\mu_{g b}\right) G_{b}-\frac{\sigma_{g b} Z_{2} G_{b}}{1+\pi_{g b} G_{b}} \\
& \frac{\mathrm{d} G_{m}}{d t}=\frac{\rho q \omega G_{b}}{1+k_{3} B}-\alpha_{3} G_{m}-\mu_{g m} G_{m} \\
& \frac{\mathrm{d} C}{d t}=\alpha_{3} G_{m}-\delta_{3} C-\mu_{c} C \\
& \frac{\mathrm{d} S_{m}}{d t}=r_{3} \delta_{3} C-a v S_{m}-\mu_{s m} S_{m} \\
& \frac{\mathrm{d} S_{h}}{d t}=a b v-\frac{\beta_{1} S_{h} H}{1+k_{1} B}-\mu_{s h} S_{h}-\frac{\sigma_{s h} Z_{1} S_{h}}{1+\pi_{s h} S_{h}} \\
& \frac{\mathrm{d} Z_{1}}{d t}=\Lambda_{z_{1}}+\left(\frac{\varepsilon_{s h} S_{h}}{1+\pi_{s h} S_{h}}+\frac{\varepsilon_{i h} I_{h}}{1+\pi_{i h} I_{h}}+\frac{\varepsilon_{t h} T_{h}}{1+\pi_{t h} T_{h}}\right) Z_{1}-\mu_{z_{1}} Z_{1} \\
& \frac{\mathrm{d} Z_{2}}{d t}=\Lambda_{z_{2}}+\left(\frac{\varepsilon_{m} M}{1+\pi_{m} M}+\frac{\varepsilon_{i r} I_{r}}{1+\pi_{i r} I_{r}}+\frac{\varepsilon_{t r} T_{r}}{1+\pi_{t r} T_{r}}+\frac{\varepsilon_{g b} G_{b}}{1+\pi_{g b} G_{b}}\right) Z_{2}-\mu_{z_{2}} Z_{2} \\
& \frac{\mathrm{d} B}{d t}=\eta_{1} \frac{S_{h} Z_{1}}{1+\pi_{s h} S_{h}}+\eta_{2} \frac{M Z_{2}}{1+\pi_{m} M}-\mu_{b} B
\end{aligned}
$$

\section{Analysis of the Model}

\subsection{Wellposedness of the model}

In this section we assess the wellposedness of the model by investigating the existence and feasibility of its solution. That is, to test whether the solutions are epidemiologically (variables have biological interpretation) and mathematically (a unique bounded solution exists for all the time) wellposed. The model system (1a)-(1o) can be expressed in the compact form $[11,19]$

$$
\frac{\mathrm{d} X}{d t}=A(x) X+F
$$

where

$$
X=\left(H, I_{h}, T_{h}, M, R, I_{r}, T_{r}, G_{b}, G_{m}, C, S_{m}, S_{h}, Z_{1}, Z_{2}, B\right)^{T},
$$




$$
A(x)=\left[\begin{array}{ccccccccccccccc}
-a_{1} & 0 & 0 & 0 & 0 & 0 & 0 & 0 & 0 & 0 & 0 & 0 & 0 & 0 & 0 \\
a_{2} & -a_{3} & 0 & 0 & 0 & 0 & 0 & 0 & 0 & 0 & 0 & 0 & 0 & 0 & 0 \\
0 & \alpha_{1} & -a_{4} & 0 & 0 & 0 & 0 & 0 & 0 & 0 & 0 & 0 & 0 & 0 & 0 \\
0 & 0 & a_{5} & -a_{6} & 0 & 0 & a_{7} & 0 & 0 & 0 & 0 & 0 & 0 & 0 & 0 \\
0 & 0 & 0 & 0 & -a_{8} & 0 & 0 & 0 & 0 & 0 & 0 & 0 & 0 & 0 & 0 \\
0 & 0 & 0 & 0 & a_{9} & -a_{10} & 0 & 0 & 0 & 0 & 0 & 0 & 0 & 0 & 0 \\
0 & 0 & 0 & 0 & 0 & \alpha_{2} & -a_{11} & 0 & 0 & 0 & 0 & 0 & 0 & 0 & 0 \\
0 & 0 & 0 & 0 & 0 & 0 & a_{12} & -a_{13} & 0 & 0 & 0 & 0 & 0 & 0 & 0 \\
0 & 0 & 0 & 0 & 0 & 0 & 0 & a_{14} & -a_{15} & 0 & 0 & 0 & 0 & 0 & 0 \\
0 & 0 & 0 & 0 & 0 & 0 & 0 & 0 & \alpha_{3} & -a_{16} & 0 & 0 & 0 & 0 & 0 \\
0 & 0 & 0 & 0 & 0 & 0 & 0 & 0 & 0 & r_{3} \delta_{3} & -a_{17} & 0 & 0 & 0 & 0 \\
0 & 0 & 0 & 0 & 0 & 0 & 0 & 0 & 0 & 0 & 0 & -a_{18} & 0 & 0 & 0 \\
0 & a_{19} & a_{20} & 0 & 0 & 0 & 0 & 0 & 0 & 0 & 0 & a_{21} & -\mu_{z_{1}} & 0 & 0 \\
0 & 0 & 0 & a_{22} & 0 & a_{23} & a_{24} & a_{25} & 0 & 0 & 0 & 0 & 0 & -\mu_{z_{2}} & 0 \\
0 & 0 & 0 & a_{26} & 0 & 0 & 0 & 0 & 0 & 0 & 0 & a_{27} & 0 & 0 & -\mu_{b}
\end{array}\right]
$$

where

$$
\begin{aligned}
& a_{1}= \frac{\beta_{1} S_{h}}{1+k_{1} B}+\mu_{h}, a_{2}=\frac{\beta_{1} S_{h}}{1+k_{1} B}, a_{3}=\alpha_{1}+\mu_{i h}+\frac{\sigma_{i h} Z_{1}}{1+\pi_{i h} I_{h}}, a_{4}=\delta_{1}+\mu_{t h}+\frac{\sigma_{t h} Z_{1}}{1+\pi_{t h} T_{h}}, \\
& a_{5}= \frac{r_{1} \delta_{1}}{1+c_{1} Z_{1}}, a_{6}=\mu_{m}+\frac{\beta_{2} R}{1+k_{2} B}+\frac{\sigma_{m} Z_{2}}{1+\pi_{m} M}, a_{7}=\frac{p r_{2} \delta_{2}}{1+c_{2} Z_{2}}, a_{8}=\frac{\beta_{2} M}{1+k_{2} B}+\mu_{r}, \\
& a_{9}= \frac{\beta_{2} M}{1+k_{2} B}, a_{10}=\alpha_{2}+\mu_{i r}+\frac{\sigma_{i r} Z_{2}}{1+\pi_{i r} I_{r}}, a_{11}=\delta_{2}+\mu_{t r}+\frac{\sigma_{t r} Z_{2}}{1+\pi_{t r} T_{r}}, a_{12}=\frac{(1-p) r_{2} \delta_{2}}{1+c_{2} Z_{2}}, \\
& a_{13}=q \omega+\mu_{g b}+\frac{\sigma_{g b} Z_{2}}{1+\pi_{g b} G_{b}}, a_{14}=\frac{\rho q \omega}{1+k_{3} B}, a_{15}=\alpha_{3}+\mu_{g m}, a_{16}=\delta_{3}+\mu_{c}, a_{17}=a v+\mu_{s m}, \\
& a_{18}=\frac{\beta_{1} H}{1+k_{1} B}+\mu_{s h}+\frac{\sigma_{s h} Z_{1}}{1+\pi_{s h} S_{h}}, a_{19}=\frac{\varepsilon_{i h} Z_{1}}{1+\pi_{i h} I_{h}}, a_{20}=\frac{\varepsilon_{t h} Z_{1}}{1+\pi_{t h} T_{h}}, a_{21}=\frac{\varepsilon_{s h} Z_{1}}{1+\pi_{s h} S_{h}}, \\
& a_{22}=\frac{\varepsilon_{m} Z_{2}}{1+\pi_{m} M}, a_{23}=\frac{\varepsilon_{i r} Z_{2}}{1+\pi_{i r} I_{r}}, a_{24}=\frac{\varepsilon_{t r} Z_{2}}{1+\pi_{t r} T_{r}}, a_{25}=\frac{\varepsilon_{g b} Z_{2}}{1+\pi_{g b} G_{b}}, a_{26}=\frac{\eta_{2} M Z_{2}}{1+\pi_{m} M}, \\
& a_{27}=\frac{\eta_{1} S_{h} Z_{1}}{1+\pi_{s h} S_{h}}
\end{aligned}
$$

and $F$ is a column vector given by

$$
F=\left(\Lambda_{h}, 0,0,0, \Lambda_{r}, 0,0,0,0,0,0, a b v, \Lambda_{z_{1}}, \Lambda_{z_{2}}, 0\right)^{T}
$$

It is observed that $A(x)$ is Meltzer matrix since all its off diagonal elements are non negative, for $x \in \mathbb{R}_{+}^{15}$ and $F \geq 0$. Therefore, the system (1a)-(1o) is positively invariant in $\mathbb{R}_{+}^{15}$, meaning that an arbitrary trajectory of the sytstem started in $\mathbb{R}_{+}^{15}$ remains there forever. Also $F$ is Lipschitz continous. Hence, a unique maximal solution exists and so

$$
\mathscr{D}=\left\{\left(H, I_{h}, T_{h}, M, R, I_{r}, T_{r}, G_{b}, G_{m}, C, S_{m}, S_{h}, Z_{1}, Z_{2}, B\right) \geq 0 \in \mathbb{R}_{+}^{15}\right\}
$$

is the feasible region for the model. Thus, the model (1a)-(1o) is epidemiologically and mathematically wellposed in the region $\mathscr{D}$. 


\subsection{Malaria Free Equilibrium (MEF) and Basic Reproduction Number, $\mathscr{R}_{0}$}

In absence of malaria infection, all variables representing infectious classes are zeros. Thus the MFE of the system (1a)(10) is

$$
E^{0}=\left(\frac{\Lambda_{h}}{\mu_{h}}, 0,0, \frac{\Lambda_{r}}{\mu_{r}}, 0,0,0,0,0,0,0, \frac{\Lambda_{z_{1}}}{\mu_{z_{1}}}, \frac{\Lambda_{z_{2}}}{\mu_{z_{2}}}, 0\right)
$$

We calculate basic reproduction number, $\mathscr{R}_{0}$ for the model (1a)-(1o) using the next generation matrix method [21]. In this method, $\mathscr{R}_{0}$ is given by the spectral radius $\rho\left(F V^{-1}\right)$, of next generation matrix $F V^{-1}$ where $F$ and $V$ are transmission and transition matrices given by

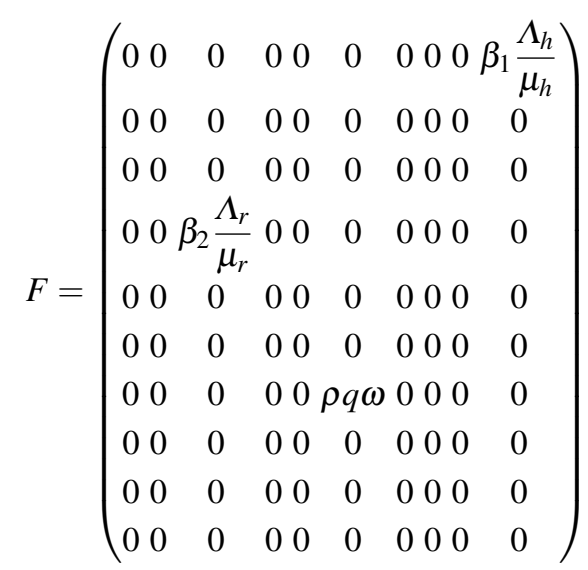

and

$$
V=\left(\begin{array}{cccccccccc}
v_{1} & 0 & 0 & 0 & 0 & 0 & 0 & 0 & 0 & 0 \\
-\alpha_{1} & v_{2} & 0 & 0 & 0 & 0 & 0 & 0 & 0 & 0 \\
0 & -v_{3} & v_{4} & 0 & -v_{5} & 0 & 0 & 0 & 0 & 0 \\
0 & 0 & 0 & v_{6} & 0 & 0 & 0 & 0 & 0 & 0 \\
0 & 0 & 0 & -\alpha_{2} & v_{7} & 0 & 0 & 0 & 0 & 0 \\
0 & 0 & 0 & 0 & -v_{8} & v_{9} & 0 & 0 & 0 & 0 \\
0 & 0 & 0 & 0 & 0 & 0 & v_{10} & 0 & 0 & 0 \\
0 & 0 & 0 & 0 & 0 & 0 & -\alpha_{3} & v_{11} & 0 & 0 \\
0 & 0 & 0 & 0 & 0 & 0 & 0 & -v_{12} & v_{13} & 0 \\
0 & 0 & 0 & 0 & 0 & 0 & 0 & 0 & 0 & v_{14}
\end{array}\right)
$$

respectively, where

$$
\begin{aligned}
v_{1} & =\alpha_{1}+\mu_{i h}+\frac{\sigma_{i h} \Lambda_{z_{1}}}{\mu_{z_{1}}}, v_{2}=\delta_{1}+\mu_{t h}+\frac{\sigma_{t h} \Lambda_{z_{1}}}{\mu_{z_{1}}}, v_{3}=\frac{r_{1} \delta_{1} \mu_{z_{1}}}{\mu_{z_{1}}+c_{1} \Lambda_{z_{1}}}, v_{4}=\beta_{2} \frac{\Lambda_{r}}{\mu_{r}}+\frac{\sigma_{m} \Lambda_{z_{2}}}{\mu_{z_{2}}}+\mu_{m} \\
v_{5} & =\frac{p r_{2} \delta_{2} \mu_{z_{2}}}{\mu_{z_{2}}+c_{2} \Lambda_{z_{2}}}, v_{6}=\alpha_{2}+\mu_{i r}+\frac{\sigma_{i r} \Lambda_{z_{2}}}{\mu_{z_{2}}}, v_{7}=\delta_{2}+\frac{\sigma_{t r} \Lambda_{z_{2}}}{\mu_{z_{2}}}+\mu_{t r}, v_{8}=\frac{(1-p) r_{2} \delta_{2} \mu_{z_{2}}}{\mu_{z_{2}}+c_{2} Z_{2}}, \\
v_{9} & =q \omega+\mu_{g b}+\frac{\sigma_{g b} \Lambda_{z_{2}}}{\mu_{z_{2}}}, v_{10}=\alpha_{3}+\mu_{g m}, v_{11}=\delta_{3}+\mu_{c}, v_{12}=r_{3} \delta_{3}, v_{13}=a v+\mu_{s m}, \\
v_{14} & =\beta_{1} \frac{\Lambda_{h}}{\mu_{h}}+\frac{\sigma_{s h} \Lambda_{z_{1}}}{\mu_{z_{1}}}+\mu_{s h}
\end{aligned}
$$


From (4) we obtained the inverse, $V^{-1}$, of $V$ given by

$$
V^{-1}=\left(\begin{array}{cccccccccc}
\frac{1}{v_{1}} & 0 & 0 & 0 & 0 & 0 & 0 & 0 & 0 & 0 \\
\frac{\alpha_{1}}{v_{2} v_{1}} & \frac{1}{v_{3} \alpha_{1}} & 0 & 0 & 0 & 0 & 0 & 0 & 0 & 0 \\
\frac{v_{3}}{v_{4} v_{2} v_{1}} & \frac{1}{v_{4} v_{2}} & \frac{v_{4}}{v_{4}} & \frac{v_{5}}{v_{7} v_{6} v_{4}} & \frac{v_{5}}{v_{7} v_{4}} & 0 & 0 & 0 & 0 & 0 \\
0 & 0 & 0 & \frac{1}{v_{6}} & 0 & 0 & 0 & 0 & 0 & 0 \\
0 & 0 & 0 & \frac{\alpha_{2}}{v_{7} v_{6}} & \frac{1}{v_{7}} & 0 & 0 & 0 & 0 & 0 \\
0 & 0 & 0 & \frac{\alpha_{2} v_{8}}{v_{9} v_{7} v_{6}} & \frac{v_{8}}{v_{9} v_{7}} & \frac{1}{v_{9}} & 0 & 0 & 0 & 0 \\
0 & 0 & 0 & 0 & 0 & 0 & \frac{1}{v_{10}} & 0 & 0 & 0 \\
0 & 0 & 0 & 0 & 0 & 0 & \frac{\alpha_{3}}{v_{11} v_{10}} & \frac{1}{v_{11}} & 0 & 0 \\
0 & 0 & 0 & 0 & 0 & 0 & \frac{v_{12} \alpha_{3}}{v_{13} v_{11} v_{10}} & \frac{v_{12}}{v_{113} v_{11}} & \frac{1}{v_{13}} & 0 \\
0 & 0 & 0 & 0 & 0 & 0 & 0 & 0 & 0 & \frac{1}{v_{14}}
\end{array}\right)
$$

Hence, from (3) and (6), we have

$$
\begin{aligned}
& F V^{-1}=\left(\begin{array}{cccccccccc}
0 & 0 & 0 & 0 & 0 & 0 & 0 & 0 & 0 & A_{1} \\
0 & 0 & 0 & 0 & 0 & 0 & 0 & 0 & 0 & 0 \\
0 & 0 & 0 & 0 & 0 & 0 & 0 & 0 & 0 & 0 \\
A_{2} & A_{3} & A_{4} & A_{5} & A_{6} & 0 & 0 & 0 & 0 & 0 \\
0 & 0 & 0 & 0 & 0 & 0 & 0 & 0 & 0 & 0 \\
0 & 0 & 0 & 0 & 0 & 0 & 0 & 0 & 0 & 0 \\
0 & 0 & 0 & 0 & 0 & 0 & 0 & 0 & 0 & 0 \\
0 & 0 & 0 & A_{7} & A_{8} & A_{9} & 0 & 0 & 0 & 0 \\
0 & 0 & 0 & 0 & 0 & 0 & 0 & 0 & 0 & 0 \\
0 & 0 & 0 & 0 & 0 & 0 & 0 & 0 & 0 & 0
\end{array}\right) \\
& A_{1}=\frac{\beta_{1} \Lambda_{h}}{v_{14} \mu_{h}}, A_{2}=\frac{\beta_{2} \alpha_{1} v_{3} \Lambda_{r}}{v_{4} v_{2} v_{1} \mu_{r}}, A_{3}=\frac{\beta_{2} v_{3} \Lambda_{r}}{v_{4} v_{2} \mu_{r}}, A_{4}=\frac{\beta_{2} \Lambda_{r}}{v_{4} \mu_{r}}, A_{5}=\frac{\beta_{2} \Lambda_{r} v_{5} \alpha_{2}}{v_{7} v_{6} v_{4} \mu_{r}}, \\
& A_{6}=\frac{\beta_{2} v_{5} \Lambda_{r}}{v_{7} v_{4} \mu_{r}}, A_{7}=\frac{\rho q \omega v_{8} \alpha_{2}}{v_{9} v_{7} v_{6}}, A_{8}=\frac{\rho q \omega v_{8}}{v_{9} v_{7}}, A_{9}=\frac{\rho q \omega}{v_{9}}
\end{aligned}
$$

The basic reproduction number, $\mathscr{R}_{01}$, is the dorminant eigenvalue of $F_{1} V_{1}^{-1}$. Hence using equations (7) and (8), we obtain only one nonzero eigenvalue which is $A_{5}$. Thus, the basic reproduction number, $\mathscr{R}_{0}$ is given by

$$
\mathscr{R}_{0}=\left[\frac{\beta_{2} r_{0}}{\beta_{2} r_{0}+\sigma_{m} z_{0}+\mu_{m}}\right]\left[\frac{\alpha_{2}}{\alpha_{2}+\mu_{i r}+\sigma_{i r} z_{0}}\right]\left[\frac{1}{\delta_{2}+\mu_{t r}+\sigma_{t r} z_{0}}\right]\left[\frac{p r_{2} \delta_{2}}{1+c_{2} z_{0}}\right]
$$

where $r_{0}=\frac{\Lambda_{r}}{\mu_{r}}$ and $z_{0}=\frac{\Lambda_{z_{2}}}{\mu_{z_{2}}}$ are values of $R$ and $Z_{2}$ at malaria-free equilibrium, respectively.

From equation (9), the term $\left(\frac{\beta_{2} r_{0}}{\beta_{2} r_{0}+\sigma_{m} z_{0}+\mu_{m}}\right)$ is the proportion of RBCs that a merozite introduced into entirely susceptile RBC population infects before it dies (either naturally or cleared by immune cells), while the term 
$\left(\frac{\alpha_{2}}{\alpha_{2}+\mu_{i r}+\sigma_{i r} z_{0}}\right)$ represents the proportion of infected RBCs that progress to schizonts before dying and the term $\left(\frac{1}{\delta_{2}+\mu_{t r}+\sigma_{t r} z_{0}}\right)$ is an average duration a schizont spends before it burst or cleared by immune cells and $\left(\frac{p r_{2} \delta_{2}}{1+c_{2} z_{0}}\right)$ is number of merozoites produced by a schizont when it bursts.

\subsection{Local Stability of MFE}

The Jacobian matrix of the system (1a)-(10) evaluated at malaria-free equilibrium, $E^{0}$, is

$$
J\left(E^{0}\right)=\left[\begin{array}{ccccccccccccccc}
-\mu_{h} & 0 & 0 & 0 & 0 & 0 & 0 & 0 & 0 & 0 & 0 & -u_{1} & 0 & 0 & 0 \\
0 & -u_{2} & 0 & 0 & 0 & 0 & 0 & 0 & 0 & 0 & 0 & u_{1} & 0 & 0 & 0 \\
0 & \alpha_{1} & -u_{3} & 0 & 0 & 0 & 0 & 0 & 0 & 0 & 0 & 0 & 0 & 0 & 0 \\
0 & 0 & u_{4} & -u_{5} & 0 & 0 & u_{6} & 0 & 0 & 0 & 0 & 0 & 0 & 0 & 0 \\
0 & 0 & 0 & -u_{7} & -\mu_{r} & 0 & 0 & 0 & 0 & 0 & 0 & 0 & 0 & 0 & 0 \\
0 & 0 & 0 & u_{7} & 0 & -u_{8} & 0 & 0 & 0 & 0 & 0 & 0 & 0 & 0 & 0 \\
0 & 0 & 0 & 0 & 0 & \alpha_{2} & -u_{9} & 0 & 0 & 0 & 0 & 0 & 0 & 0 & 0 \\
0 & 0 & 0 & 0 & 0 & 0 & u_{10} & -u_{11} & 0 & 0 & 0 & 0 & 0 & 0 & 0 \\
0 & 0 & 0 & 0 & 0 & 0 & 0 & \rho q \omega & -u_{12} & 0 & 0 & 0 & 0 & 0 & 0 \\
0 & 0 & 0 & 0 & 0 & 0 & 0 & 0 & \alpha_{3} & -u_{13} & 0 & 0 & 0 & 0 & 0 \\
0 & 0 & 0 & 0 & 0 & 0 & 0 & 0 & 0 & r_{3} \delta_{3} & -u_{14} & 0 & 0 & 0 & 0 \\
0 & 0 & 0 & 0 & 0 & 0 & 0 & 0 & 0 & 0 & 0 & -u_{15} & 0 & 0 & 0 \\
0 & u_{16} & u_{17} & 0 & 0 & 0 & 0 & 0 & 0 & 0 & 0 & u_{18} & -\mu_{z_{1}} & 0 & 0 \\
0 & 0 & 0 & u_{19} & 0 & u_{20} & u_{21} & u_{22} & 0 & 0 & 0 & 0 & 0 & -\mu_{z_{2}} & 0 \\
0 & 0 & 0 & u_{23} & 0 & 0 & 0 & 0 & 0 & 0 & 0 & u_{24} & 0 & 0 & -\mu_{b}
\end{array}\right]
$$

where

$$
\begin{aligned}
& u_{1}=\beta_{1} \frac{\Lambda_{h}}{\mu_{h}}, u_{2}=\alpha_{1}+\mu_{i h}+\sigma_{i h} \frac{\Lambda_{z_{1}}}{\mu_{z_{1}}}, u_{3}=\delta_{1}+\mu_{t h}+\sigma_{t h} \frac{\Lambda_{z_{1}}}{\mu_{z_{1}}}, u_{4}=\frac{r_{1} \delta_{1} \mu_{z_{1}}}{\mu_{z_{1}}+c_{1} \Lambda_{z_{1}}}, \\
& u_{5}=\beta_{2} \frac{\Lambda_{r}}{\mu_{r}}+\sigma_{m} \frac{\Lambda_{z_{2}}}{\mu_{z_{2}}}+\mu_{m}, u_{6}=\frac{p r_{2} \delta_{2} \mu_{z_{2}}}{\mu_{z_{2}}+c_{2} \Lambda_{z_{2}}}, u_{7}=\beta_{2} \frac{\Lambda_{r}}{\mu_{r}}, u_{8}=\mu_{i r}+\alpha_{2}+\sigma_{i r} \frac{\Lambda_{z_{2}}}{\mu_{z_{2}}} \\
& u_{9}=\delta_{2}+\mu_{t r}+\sigma_{t r} \frac{\Lambda_{z_{2}}}{\mu_{z_{2}}}, u_{10}=\frac{(1-p) r_{2} \delta_{2} \mu_{z_{2}}}{\mu_{z_{2}}+c_{2} \Lambda_{z_{2}}}, u_{11}=q \omega+\mu_{g b}+\sigma_{g b} \frac{\Lambda_{z_{2}}}{\mu_{z_{2}}}, u_{12}=\alpha_{3}+\mu_{g m}, \\
& u_{13}=\delta_{3}+\mu_{c}, u_{14}=a v+\mu_{s m}, u_{15}=\beta_{1} \frac{\Lambda_{h}}{\mu_{h}}+\sigma_{s h} \frac{\Lambda_{z_{1}}}{\mu_{z_{1}}}+\mu_{s h}, u_{16}=\varepsilon_{i h} \frac{\Lambda_{z_{1}}}{\mu_{z_{1}}}, u_{17}=\varepsilon_{t h} \frac{\Lambda_{z_{1}}}{\mu_{z_{1}}} \\
& u_{18}=\varepsilon_{s h} \frac{\Lambda_{z_{1}}}{\mu_{z_{1}}}, u_{19}=\varepsilon_{m} \frac{\Lambda_{z_{2}}}{\mu_{z_{2}}}, u_{20}=\varepsilon_{i r} \frac{\Lambda_{z_{2}}}{\mu_{z_{2}}}, u_{21}=\varepsilon_{t r} \frac{\Lambda_{z_{2}}}{\mu_{z_{2}}}, u_{22}=\varepsilon_{g b} \frac{\Lambda_{z_{2}}}{\mu_{z_{2}}}, u_{22}=\varepsilon_{s h} \frac{\Lambda_{z_{1}}}{\mu_{z_{1}}} \\
& u_{23}=\eta_{2} \frac{\Lambda_{z_{2}}}{\pi_{m} \mu_{z_{2}}}, u_{24}=\eta_{1} \frac{\Lambda_{z_{1}}}{\pi_{s h} \mu_{z_{1}}}
\end{aligned}
$$

Local stability of a MFE, $E^{0}$, is determined by using the signs of real part of eigenvalues of $J\left(E^{0}\right)$. The MFE is locally assympotically stable if and only if all eigenvalues of $J\left(E^{0}\right)$ have negative real parts. Clearly, six eigenvalues $-\mu_{h},-\mu_{r},-u_{14},-\mu_{z_{1}},-\mu_{z_{2}}$ and $-\mu_{b}$ are negative. The other nine eigenvalues are obtained from a reduced $9 \times 9$ submatrix, $J_{1}\left(E^{0}\right)$, given by 


$$
J_{1}\left(E^{0}\right)=\left[\begin{array}{ccccccccc}
-u_{2} & 0 & 0 & 0 & 0 & 0 & 0 & 0 & u_{1} \\
\alpha_{1} & -u_{3} & 0 & 0 & 0 & 0 & 0 & 0 & 0 \\
0 & u_{4} & -u_{5} & 0 & u_{6} & 0 & 0 & 0 & 0 \\
0 & 0 & u_{7} & -u_{8} & 0 & 0 & 0 & 0 & 0 \\
0 & 0 & 0 & \alpha_{2} & -u_{9} & 0 & 0 & 0 & 0 \\
0 & 0 & 0 & 0 & u_{10} & -u_{11} & 0 & 0 & 0 \\
0 & 0 & 0 & 0 & 0 & \rho q \omega & -u_{12} & 0 & 0 \\
0 & 0 & 0 & 0 & 0 & 0 & \alpha_{3} & -u_{13} & 0 \\
0 & 0 & 0 & 0 & 0 & 0 & 0 & 0 & -u_{15}
\end{array}\right]
$$

From eighth column of $J_{1}\left(E^{0}\right)$ we observe that other eigenvalue is $-u_{13}$ which is also negative. Further reduction of this, leads us to $8 \times 8$ submatrix given by

$$
J_{2}\left(E^{0}\right)=\left[\begin{array}{cccccccc}
-u_{2} & 0 & 0 & 0 & 0 & 0 & 0 & u_{1} \\
\alpha_{1} & -u_{3} & 0 & 0 & 0 & 0 & 0 & 0 \\
0 & u_{4} & -u_{5} & 0 & u_{6} & 0 & 0 & 0 \\
0 & 0 & u_{7} & -u_{8} & 0 & 0 & 0 & 0 \\
0 & 0 & 0 & \alpha_{2} & -u_{9} & 0 & 0 & 0 \\
0 & 0 & 0 & 0 & u_{10} & -u_{11} & 0 & 0 \\
0 & 0 & 0 & 0 & 0 & \rho q \omega & -u_{12} & 0 \\
0 & 0 & 0 & 0 & 0 & 0 & 0 & -u_{15}
\end{array}\right]
$$

from which another eigenvalue $-u_{12}$ is obtained. Another reduction leads a $7 \times 7$ submatrix given

$$
J_{3}\left(E^{0}\right)=\left[\begin{array}{ccccccc}
-u_{2} & 0 & 0 & 0 & 0 & 0 & u_{1} \\
\alpha_{1} & -u_{3} & 0 & 0 & 0 & 0 & 0 \\
0 & u_{4} & -u_{5} & 0 & u_{6} & 0 & 0 \\
0 & 0 & u_{7} & -u_{8} & 0 & 0 & 0 \\
0 & 0 & 0 & \alpha_{2} & -u_{9} & 0 & 0 \\
0 & 0 & 0 & 0 & u_{10} & -u_{11} & 0 \\
0 & 0 & 0 & 0 & 0 & 0 & -u_{15}
\end{array}\right]
$$

And from $J_{3}\left(E^{0}\right)$ above we obtained another eigenvalue $-u_{11}$, and a new reduced matrix is

$$
J_{4}\left(E^{0}\right)=\left[\begin{array}{cccccc}
-u_{2} & 0 & 0 & 0 & 0 & u_{1} \\
\alpha_{1} & -u_{3} & 0 & 0 & 0 & 0 \\
0 & u_{4} & -u_{5} & 0 & u_{6} & 0 \\
0 & 0 & u_{7} & -u_{8} & 0 & 0 \\
0 & 0 & 0 & \alpha_{2} & -u_{9} & 0 \\
0 & 0 & 0 & 0 & 0 & -u_{15}
\end{array}\right]
$$

We investigate the signs of other remaining six eigenvalues using the trace-determinant technique. If the trace and determinat of $J_{4}\left(E^{0}\right)$ are strictly negative and positive respectively, then all eigenvalues of $J_{4}\left(E^{0}\right)$ have negative real parts. The following results were obtained using MAPLE 12,

$$
\operatorname{tr}\left(J_{4}\left(E^{0}\right)\right)=-\left[u_{2}+u_{3}+u_{5}+u_{8}+u_{9}+u_{15}\right]<0
$$


and

$$
\operatorname{det}\left(J_{4}\left(E^{0}\right)\right)=\left(u_{2} u_{3} u_{15} u_{5} u_{8} u_{9}\right)\left(1-\mathscr{R}_{0}\right)>0
$$

where

$$
\mathscr{R}_{0}=\frac{\alpha_{2} u_{6} u_{7}}{u_{5} u_{8} u_{9}}
$$

since the values of $u^{\prime}$ s given in equation (10) are all positive, then equation (12) is true only if

$$
\mathscr{R}_{0}<1
$$

Thus, all eigenvalues of $J\left(E^{0}\right)$ have negative real parts only $\mathscr{R}_{0}<1$. Hence, MFE is locally assymptotically stable provided $\mathscr{R}_{0}<1$, which leads us to the following theorem.

Theorem 1. The malaria-free equilibrium, $E^{0}$, of the model (1a)-(1o), is locally asymptotically stable when $\mathscr{R}_{0}<1$ and unstable otherwise.

\subsection{Global Stability of MFE}

We applied the Meltzer matrix theory to establish the global stability of MFE as used in [5], [17] and [19] by expressing the model (1a)-(1o) in the form

$$
\left\{\begin{array}{l}
\frac{d X_{n}}{d t}=A_{1}(x)\left(X_{n}-X_{E^{0}, n}\right)+A_{12}(x) X_{e} \\
\frac{d X_{e}}{d t}=A_{2}(x) X_{e}
\end{array}\right.
$$

where $X_{n}$ is the vector of non-transmitting classes and $X_{e}$ is the vector of transmitting classes. For our model, we have

$$
\begin{gathered}
X_{n}=\left(H, R, Z_{1}, Z_{2}, B\right) \text { and } X_{e}=\left(I_{h}, T_{h}, M, I_{r}, T_{r}, G_{b}, G_{m}, C, S_{m}, S_{h}\right) \\
X_{E^{0}, n}=\left(\frac{\Lambda_{h}}{\mu_{h}}, \frac{\Lambda_{r}}{\mu_{r}}, \frac{\Lambda_{z_{1}}}{\mu_{z_{1}}}, \frac{\Lambda_{z_{2}}}{\mu_{z_{2}}}, 0\right) \text { and } A_{1}(x)=\left(\begin{array}{cccccc}
-\mu_{h} & 0 & 0 & 0 & 0 \\
0 & -\mu_{r} & 0 & 0 & 0 \\
0 & 0 & -\mu_{z_{1}} & 0 & 0 \\
0 & 0 & 0 & -\mu_{z_{2}} & 0 \\
0 & 0 & 0 & 0 & -\mu_{b}
\end{array}\right) \\
A_{12}(x)=\left(\begin{array}{cccccccccc}
0 & 0 & 0 & 0 & 0 & 0 & 0 & 0 & 0 & -d_{1} \\
0 & 0 & -d_{2} & 0 & 0 & 0 & 0 & 0 & 0 & 0 \\
d_{3} & d_{4} & 0 & 0 & 0 & 0 & 0 & 0 & 0 & d_{5} \\
0 & 0 & d_{6} & d_{7} & d_{8} & d_{9} & 0 & 0 & 0 & 0 \\
0 & 0 & d_{10} & 0 & 0 & 0 & 0 & 0 & 0 & d_{11}
\end{array}\right)
\end{gathered}
$$

where

$$
\begin{aligned}
d_{1} & =\frac{\beta_{1} H}{1+k_{1} B}, d_{2}=\frac{\beta_{2} R}{1+k_{2} B}, d_{3}=\frac{\varepsilon_{i h} Z_{1}}{1+\pi_{i h} I_{h}}, d_{4}=\frac{\varepsilon_{t h} Z_{1}}{1+\pi_{t h} T_{h}}, d_{5}=\frac{\varepsilon_{s h} Z_{1}}{1+\pi_{s h} S_{h}}, \\
d_{6} & =\frac{\varepsilon_{m} Z_{2}}{1+\pi_{m} M}, d_{7}=\frac{\varepsilon_{i r} Z_{2}}{1+\pi_{i r} I_{r}}, d_{8}=\frac{\varepsilon_{i r} Z_{2}}{1+\pi_{t r} T_{r}}, d_{9}=\frac{\varepsilon_{g b} Z_{2}}{1+\pi_{g b} G_{b}}, d_{10}=\frac{\eta_{2} Z_{2}}{1+\pi_{m} M} \\
d_{11} & =\frac{\eta_{1} Z_{1}}{1+\pi_{s h} S_{h}}
\end{aligned}
$$


and

$$
A_{2}(x)=\left(\begin{array}{cccccccccc}
-w_{1} & 0 & 0 & 0 & 0 & 0 & 0 & 0 & 0 & w_{2} \\
\alpha_{1} & -w_{3} & 0 & 0 & 0 & 0 & 0 & 0 & 0 & 0 \\
0 & w_{4} & -w_{5} & 0 & w_{6} & 0 & 0 & 0 & 0 & 0 \\
0 & 0 & w_{7} & -w_{8} & 0 & 0 & 0 & 0 & 0 & 0 \\
0 & 0 & 0 & \alpha_{2} & -w_{9} & 0 & 0 & 0 & 0 & 0 \\
0 & 0 & 0 & 0 & w_{10} & -w_{11} & 0 & 0 & 0 & 0 \\
0 & 0 & 0 & 0 & 0 & w_{12} & -w_{13} & 0 & 0 & 0 \\
0 & 0 & 0 & 0 & 0 & 0 & \alpha_{3} & -w_{14} & 0 & 0 \\
0 & 0 & 0 & 0 & 0 & 0 & 0 & w_{15} & -w_{16} & 0 \\
0 & 0 & 0 & 0 & 0 & 0 & 0 & 0 & w_{17} & -w_{18}
\end{array}\right)
$$

where

$$
\begin{aligned}
w_{1} & =\alpha_{1}+\mu_{i h}+\frac{\sigma_{i h} Z_{1}}{1+\pi_{i h} I_{h}}, w_{2}=\frac{\beta_{1} H}{1+k_{1} B}, w_{3}=\delta_{1}+\mu_{t h}+\frac{\sigma_{t h} Z_{1}}{1+\pi_{t h} T_{h}}, w_{4}=\frac{r_{1} \delta_{1}}{1+c_{1} Z_{1}}, \\
w_{5} & =\frac{\beta_{2} R}{1+k_{2} B}+\mu_{m}+\frac{\sigma_{m} Z_{2}}{1+\pi_{m} M}, w_{6}=\frac{p r_{2} \delta_{2}}{1+c_{2} Z_{2}}, w_{7}=\frac{\beta_{2} R}{1+k_{2} B}, w_{8}=\alpha_{2}+\mu_{i r}+\frac{\sigma_{i r} Z_{2}}{1+\pi_{i r} I_{r}}, \\
w_{9} & =\delta_{2}+\mu_{t r}+\frac{\sigma_{t r} Z}{1+\pi_{t r} T_{r}}, w_{10}=\frac{(1-p) r_{2} \delta_{2}}{1+c_{2} Z_{2}}, w_{11}=q \omega+\mu_{g b}+\frac{\sigma_{g b} Z_{2}}{1+\pi_{g b} G_{b}}, w_{12}=\frac{\rho q \omega}{1+k_{3} B}, \\
w_{13} & =\alpha_{3}+\mu_{g m}, w_{14}=\delta_{3}+\mu_{c}, w_{15}=r_{3} \delta_{3}, w_{16}=a v+\mu_{s m}, w_{17}=\frac{a b v}{I_{h}}, \\
w_{18} & =\frac{\beta_{1} H}{1+k_{1} B}+\frac{\sigma_{s h} Z_{1}}{1+\pi_{s h} S_{h}}+\mu_{s h}
\end{aligned}
$$

It can easily be observed from (15) that, all eigenvalues of $A_{1}$ are real and negative.

So, the system

$$
\frac{\mathrm{d} X_{n}}{\mathrm{~d} t}=A_{1}(x)\left(X_{n}-X_{E^{01}, n}\right)+A_{12}(x) X_{e}
$$

is globally assymptotically stable at $E^{01}$. It can be observed from (18) and (19) that all off diagonal elements of $A_{2}$ are non-negative. Therefore, $A_{2}$ is a Metzler matrix. To establish the global asymptotic stability of MFE, we have to show that $A_{2}$ is Metzler stable matrix (all its diagonal elements are negative) by proving the following lemma as described in [11] and [13].

Lemma 1. Let $M$ be a square Metzler matrix written in block form $M=\left(\begin{array}{ll}M_{11} & M_{12} \\ M_{21} & M_{22}\end{array}\right)$ with $M_{11}$ and $M M_{22}$ square matrices. $M$ is Metzler stable if and only if matrices $M_{11}$ and $M_{22}-M_{21} M_{11}^{-1} M_{12}$ are Metzler stable.

From equation (18) we have $M=A_{2}$,

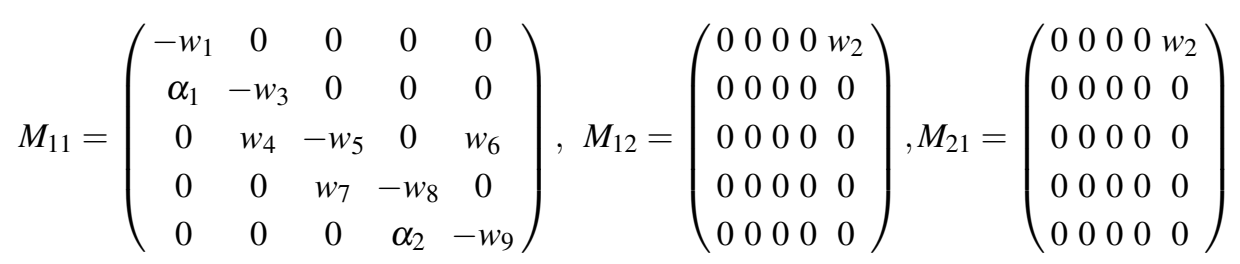


and

$$
M_{22}=\left(\begin{array}{ccccc}
-w_{11} & 0 & 0 & 0 & 0 \\
w_{12} & -w_{13} & 0 & 0 & 0 \\
0 & \alpha_{3} & -w_{14} & 0 & 0 \\
0 & 0 & w_{15} & -w_{16} & 0 \\
0 & 0 & 0 & w_{17} & -w_{18}
\end{array}\right)
$$

Hence,

$$
M_{22}-M_{21} M_{11}^{-1} M_{12}=\left(\begin{array}{ccccc}
-w_{11} & 0 & 0 & 0 & \frac{\alpha_{1} \alpha_{2} w_{10} w_{7} w_{4} w_{2}}{w_{1} w_{3}\left(w_{5} w_{8} w_{9}-\alpha_{2} w_{6} w_{7}\right)} \\
w_{12} & -w_{13} & 0 & 0 & 0 \\
0 & \alpha_{3} & -w_{14} & 0 & 0 \\
0 & 0 & w_{15} & -w_{16} & 0 \\
0 & 0 & 0 & w_{17} & -w_{18}
\end{array}\right)
$$

Clearly, $M_{11}$ is Metzler stable matrix. But, $M_{22}-M_{21} M_{11}^{-1} M_{12}$ is Metzler stable matrix only if

$$
\frac{\alpha_{1} \alpha_{2} w_{10} w_{7} w_{4} w_{2}}{w_{1} w_{3}\left(w_{5} w_{8} w_{9}-\alpha_{2} w_{6} w_{7}\right)} \geq 0
$$

It is observed that equation (20) holds only when

$$
\frac{\alpha_{2} w_{6} w_{7}}{w_{5} w_{8} w_{9}} \leq 1
$$

Substituting the expression of $w_{5}, w_{6}, w_{7}, w_{8}$ and $w_{9}$ given in equation (19) evaluated at MFE, into equation (21) we get

$$
\frac{\frac{\beta_{2} \Lambda_{r}}{\mu_{r}} \alpha_{2} \frac{p r_{2} \delta_{2} \mu_{z}}{\mu_{z}+c_{2} \Lambda_{z}}}{\left(\frac{\beta_{2} \Lambda_{r}}{\mu_{r}}+\mu_{m}+\frac{\sigma_{m} \Lambda_{z}}{\mu_{z}}\right)\left(\alpha_{2}+\mu_{i r}+\frac{\sigma_{i r} \Lambda_{z}}{\mu_{z}}\right)\left(\delta_{2}+\mu_{t r}+\frac{\sigma_{t r} \Lambda_{z}}{\mu_{z}}\right)}=\mathscr{R}_{0} \leq 1
$$

which leads us to the following theorem.

Theorem 2.A MFE, $E^{0}$ of the model (1a)-(1o) is globally assymptotically stable in $\mathscr{D}$ if $\mathscr{R}_{0} \leq 1$ and unstable if $\mathscr{R}_{0}>1$

\subsection{Existence and Stability of Malaria Infection Equilibrium}

If $\mathscr{R}_{0}>1$, then Theorem 2 suggests the existence of malaria-infection equilibrium (MIE), which is given by

$$
E^{*}=\left(H^{*}, I_{h}^{*}, T_{h}^{*}, M^{*}, R^{*}, I_{r}^{*}, T_{r}^{*}, G_{b}^{*}, G_{m}^{*}, C^{*}, S_{m}^{*}, S_{h}^{*}, Z_{1}^{*}, Z_{2}^{*}, B^{*}\right)
$$

Due to the complexity of the model system (1a)-(10), it is found awkward to express MIE explicitly. Therefore, the existence and stability are established numerically in the next section.

\section{Numerical Simulations and Discussion}

In this section, we perform some numerical simulations of the model (1a)-(1o), to illustrate the dynamics of model using MATLAB symbolic package. In the simulation of this model, initial values are almost assummed to allow computer executions, and their values are as listed in Table 3 


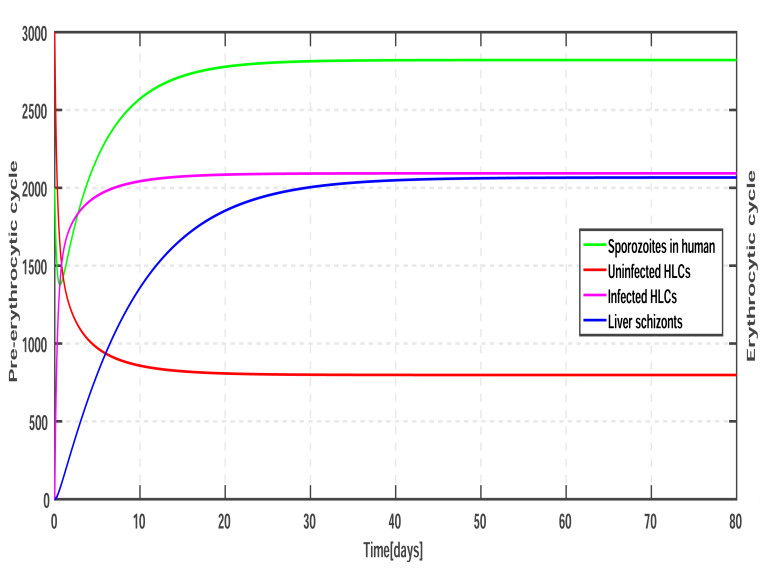

(a) Exo-erythrocytic cycle

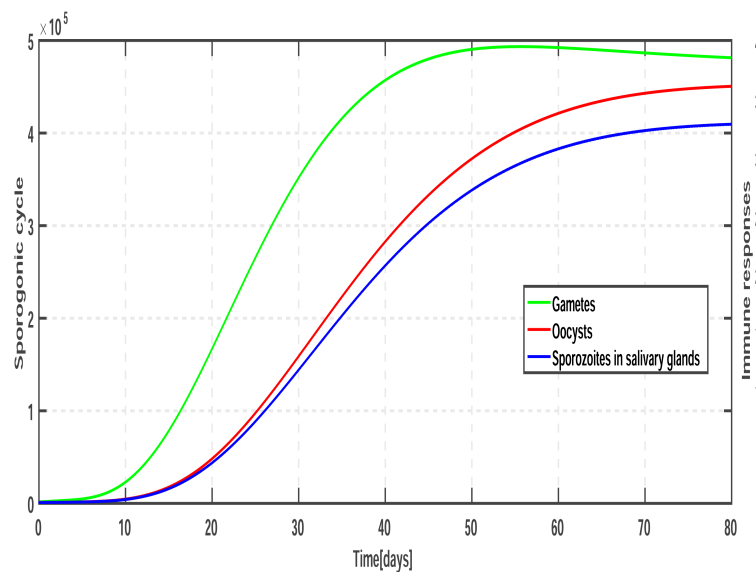

(c) Sporogonic cycle

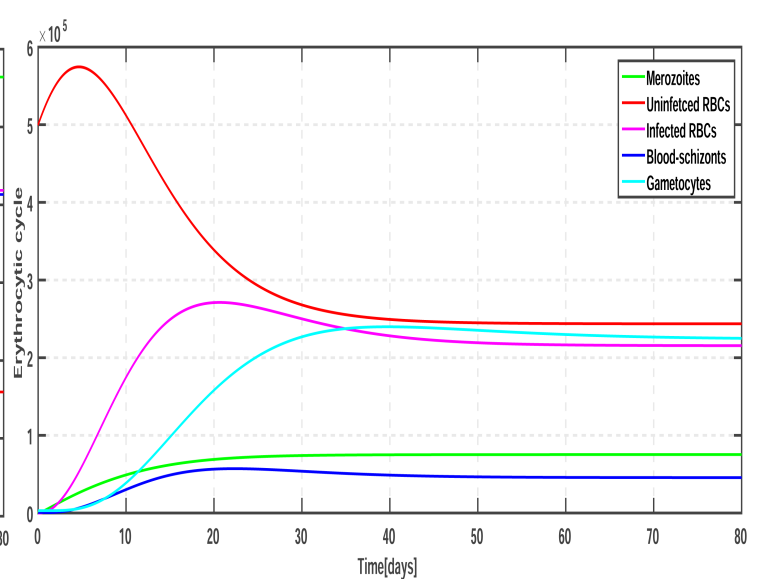

(b) Erythrocytic cycle

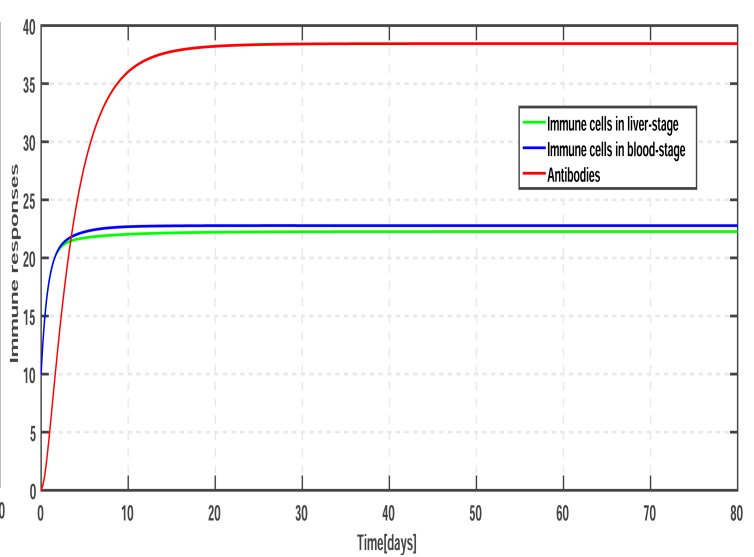

(d) Immune responses

Fig. 2: Variation of populations at Exo-erythrocytic, Erythrocytic, Sporogonic cycles and immune responses with time to verify the existence of malaria infection equilibrium.

Table 3: Initial values of variables of the model (1a)-(1o)

\begin{tabular}{|l|l|l|l|l|l|l|l|l|l|l|l|l|l|l|l|}
\hline Variable & $H$ & $I_{h}$ & $T_{h}$ & $M$ & $R$ & $I_{r}$ & $T_{r}$ & $G_{b}$ & $G_{m}$ & $C$ & $S_{m}$ & $S_{h}$ & $Z_{1}$ & $Z_{2}$ & $B$ \\
\hline \hline Initial values & 3000 & 0 & 0 & 2000 & 500000 & 0 & 1000 & 3000 & 1500 & 1000 & 2000 & 2000 & 10 & 10 & 0 \\
\hline
\end{tabular}

The numerical values of parameters used for simulation of our model are listed in Table 2. These values are either assumed or taken from some related studies among existing literature. The reason as to why some parameters values are assumed is that, mathematical modelling on liver and/or mosquito stage dynamics of malaria infection have not yet been done or the values found on existing literatures are not suitable for this model. However, our main concern is not on accuracy of these parameter values, rather is on the effect of these parameters on basic reproduction number, which alerts on how and where to target to eradicate or control the disease [6].

We performed numerical simulations to establish the existence of malaria infection equilibrium (MIE) as stated earlier. Figures $2 \mathrm{a}, 2 \mathrm{~b}, 2 \mathrm{c}$ and $2 \mathrm{~d}$ indicate that each variable varies with time and reaches a constant value (i.e., a value at MIE). Therefore, Figure 2 proves the existence of malaria-infection equilibrium, $E^{*}$ for the model (1a) -(1o). Now, let us assess 


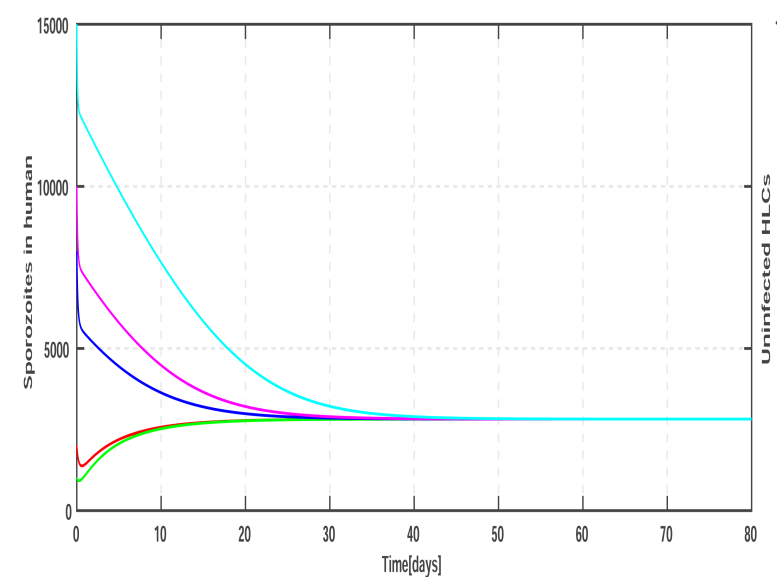

(a) $S_{h}^{0}=1000,2000,8000,10000,15000$

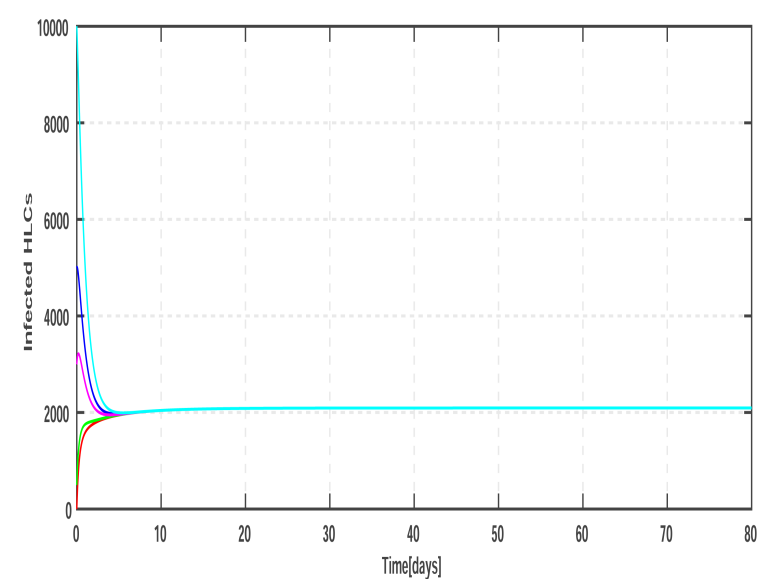

(c) $I_{h}^{0}=0,500,3000,5000,10000$

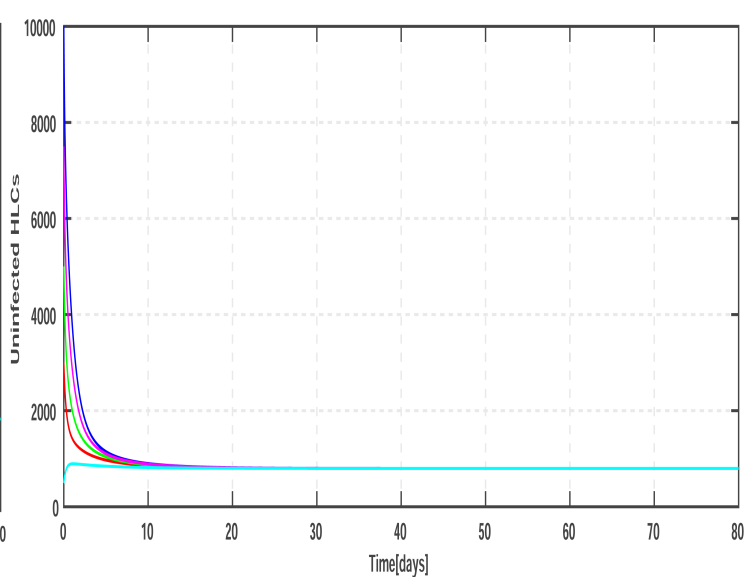

(b) $H^{0}=500,3000,5000,7500,10000$

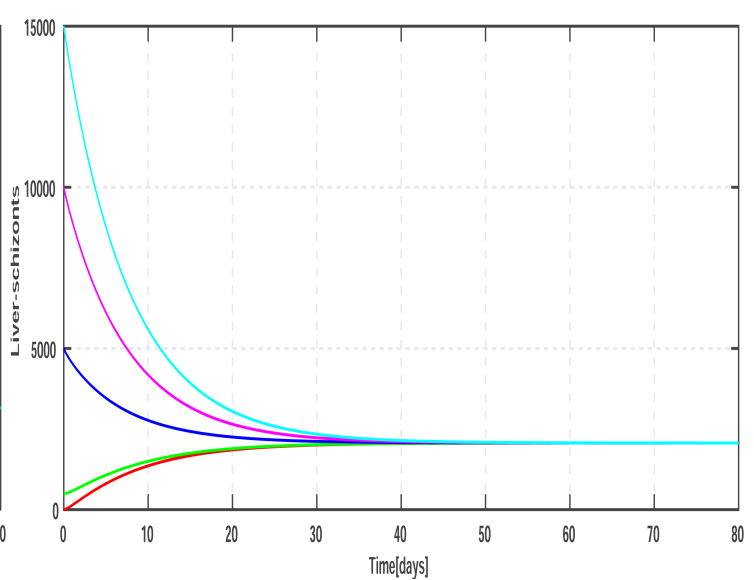

(d) $T_{h}^{0}=0,500,5000,10000,15000$

Fig. 3: Variation of populations at Exo-erythrocytic, Erythrocytic, Sporogonic cycles and immune responses with time to verify the existence of malaria infection equilibrium.

for stability of $E^{*}$. Figure 3 depicts that with different initial values, each variable in exo-erythrocytic cycle converges to certain value (value at MIE).

Figure 4 shows that each variable of erythrocytic cycle converges to a certain steady value irrespective of initial value it takes. The case is similar for variables of sporogonic cycle and for immune responses as indicated in Figure 5 and Figure 6 respectively. Therefore, with reference to Figures $3,4,5$ and 6 , we conclude that, malaria infection equilibrium, $E^{*}$ is, globally asymptotically stable.

Moreover, this model indicates promising results on the control of malaria infection at the erythrocytic stages. From Figure 7 it is observed that inclusion of immune responses in the model has impact on increasing the number uRBCs and reducing the number of iRBCs, merozoites and gametocytes. The minimum number of uRBCs to basic model (nonimmune) is below $2 \times 10^{-5}$ cells per microlitre, while in this model where effects of immune responses are included, the minimal number of uRBCs is found to be above $2 \times 10^{-5}$ cells per microlitre as indicated in Figure $7 \mathrm{a}$. On the other hand, maximal number of iRBCs in nonimmune model and in a model with immune effect are approximately to $3.4 \times 10^{-5}$ and $2.7 \times 10^{-5}$ cells per microlitre respectively as illustrated in Figure 7b. In addition to that, there is noticable decrease in number of merozoites and gametocytes (See Figures $7 \mathrm{c}$ and $7 \mathrm{~d}$ respectively). 


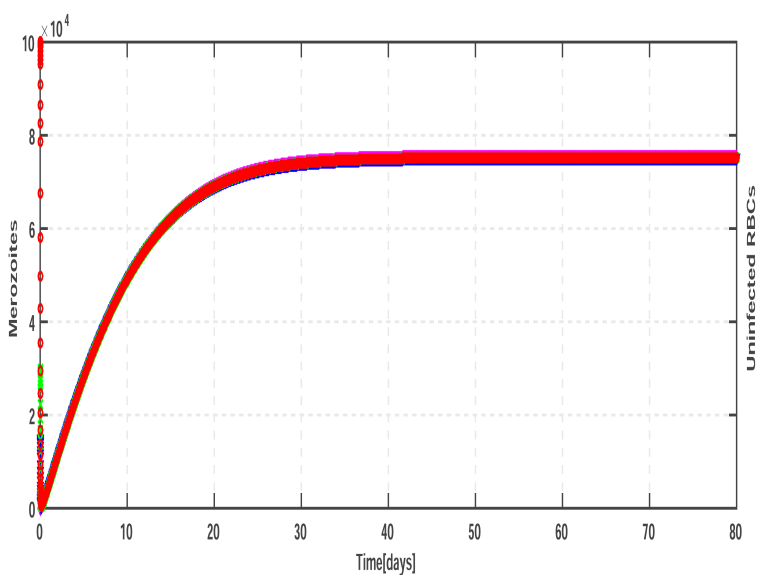

(a) $M^{0}=0,2000,15000,30000,100000$

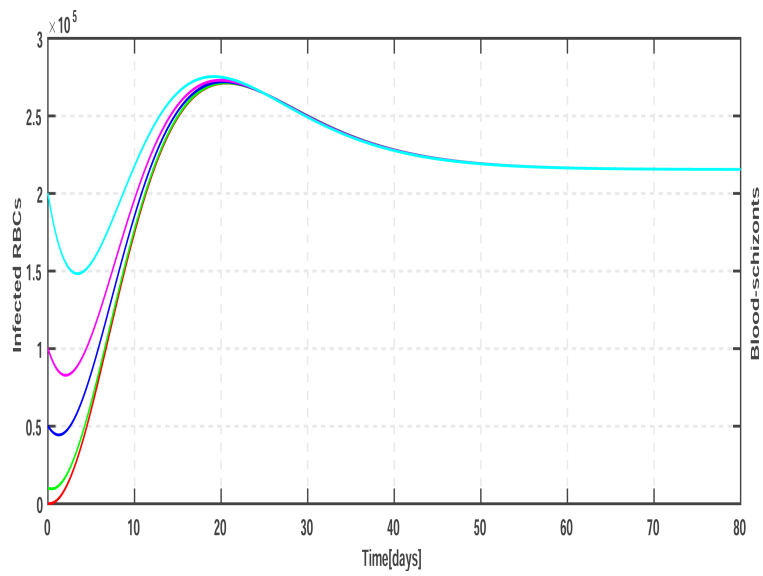

(c) $I_{r}^{0}=0,10000,50000,100000,200000$

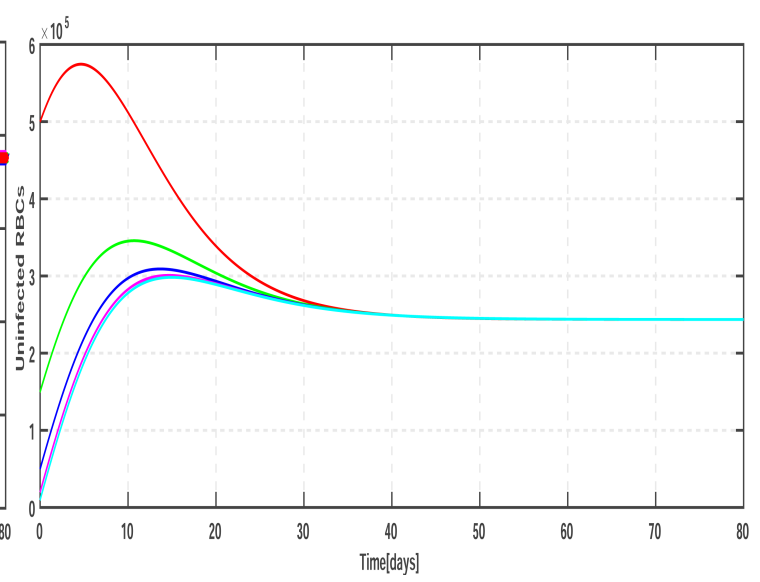

(b) $R^{0}=10000,20000,50000,150000,500000$

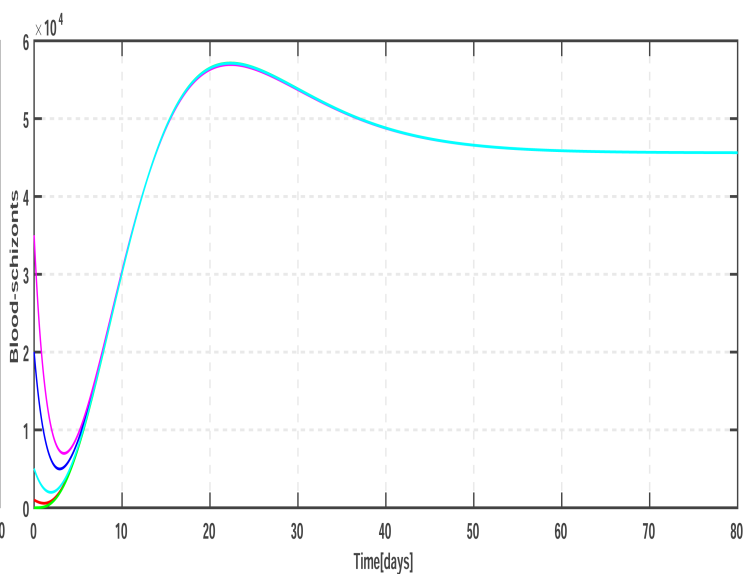

(d) $T_{r}^{0}=0,1000,5000,20000,35000$

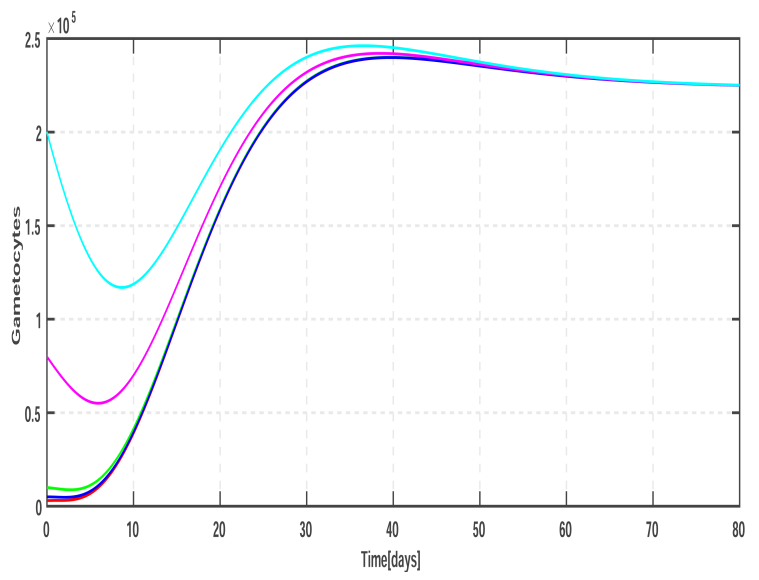

(e) $G_{b}^{0}=3000,5000,10000,80000,200000$

Fig. 4: Numerical simulation to show global stability of MIE for variables in erythrocytic cycle. 


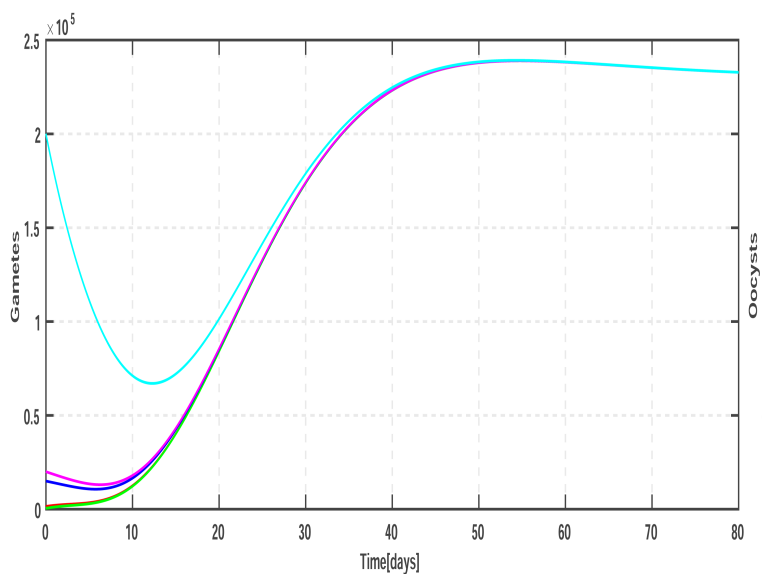

(a) $G_{m}^{0}=500,1500,3000,15000,20000$

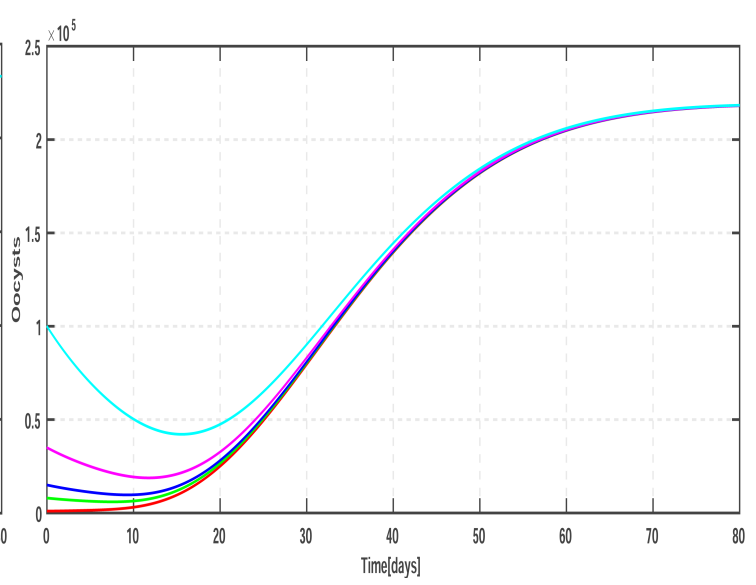

(b) $C^{0}=1000,8000,15000,35000,100,000$

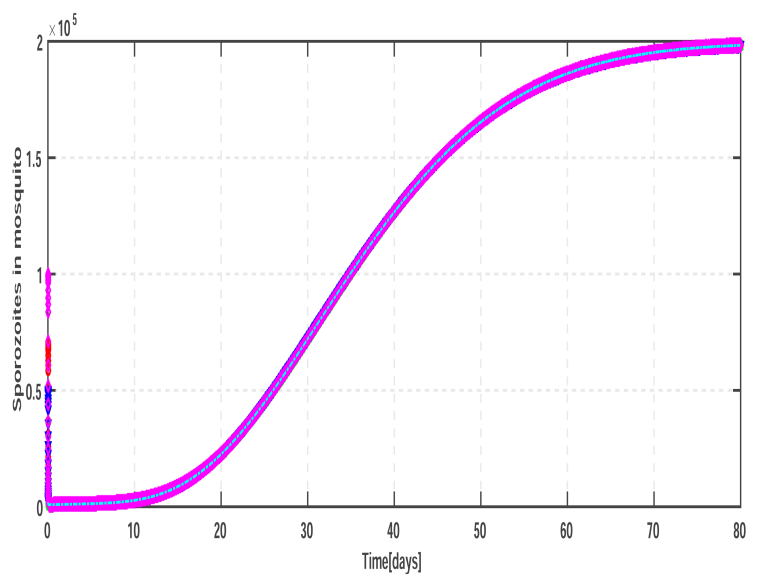

(c) $S_{m}^{0}=2000,15000,50000,7000,100,000$

Fig. 5: Numerical simulation to show global stability of MIE for variables in Sporogonic cycle.

However, the immune responses have shown to have little or almost no effect on attacking liver stage malaria infection. This is because there is unnoticable change in number of uHLCs and iHLCs even after immune system being included the model as indicated in Figure 8. It can be observed that despite of very big change on value of $k_{1}$ (efficiency of antibodies to block invasion of hepatocytes by sporozoites) from $0.075 /$ day to $0.9 /$ day, but still the change on number of uHLCs and iHLCs is almost negligible. The case is the same to sporozoites injected and hepatic schizonts. These results suggest that at the liver stage is not a good target for intervetion using immune responses.

This finding supports the [15]'s argument that the function of antibodies to sporozoites is thought to be insignificant. Furthermore, the immune responses has shown positive results on the sporogonic stages of malaria parasites as shown in Figure 9. This implies that, the antibodies taken up by mosquito during the blood meal results on lysis of gametocytes and prevent the development of parasites within mosquito. Consequently, it can lead us to a population of non-infectious mosquitoes, and reduce further mosquito-human malaria transmission.

Since the immune responses are mostly stimulated by infection and in most cases last within a short period of time, we have assessed the impact of lifespan of immune cells on the elimination (reduction) of malaria infection. The results show that the lifespan varies inversely with number of infected RBCs, merozoites and gametocytes as depicted in Figures 10b, 10c and 10d respectively. In the other hand, from Figure 10a we observed that the lifespan of immune cells has great 


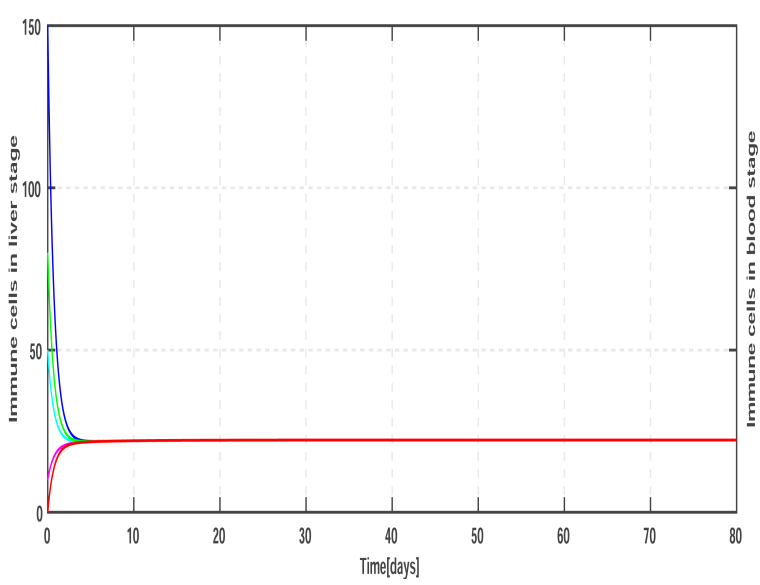

(a) $Z_{1}^{0}=0,10,50,80,150$

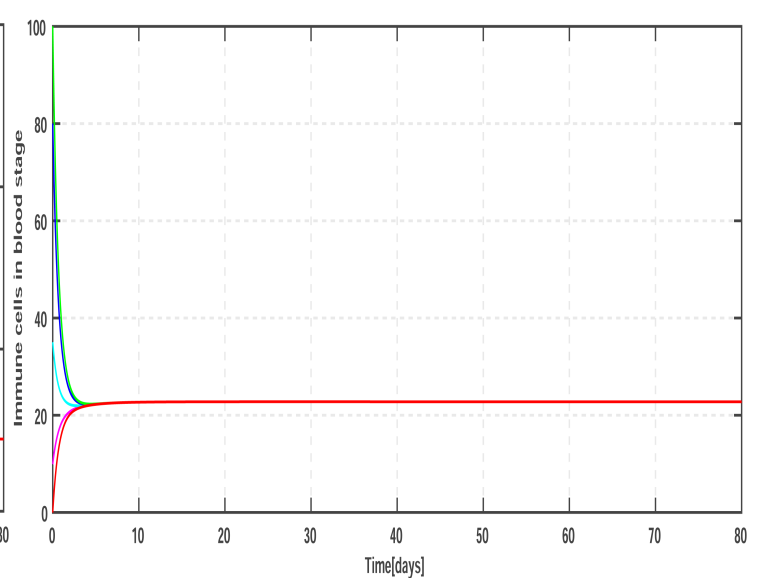

(b) $Z_{2}^{0}=0,10,35,80,100$

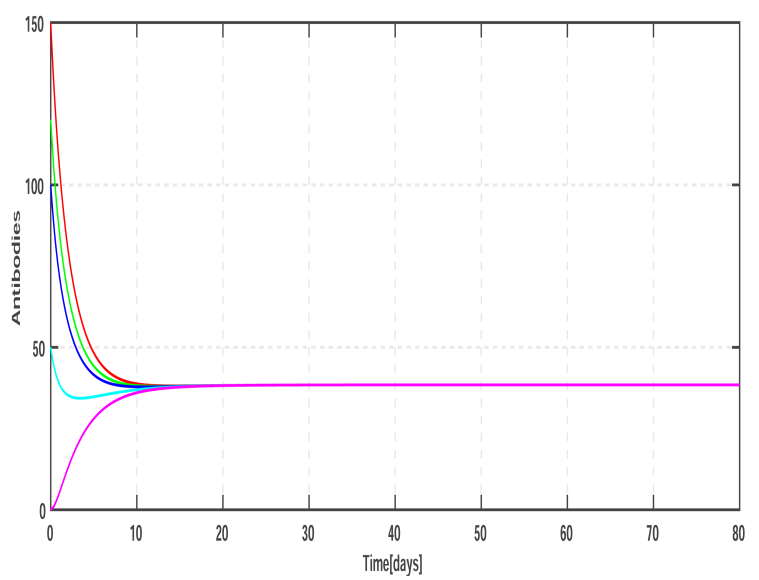

(c) $B^{0}=0,50,100,120,150$

Fig. 6: Numerical simulation to show global stability of MIE for variables for immune responses.

influence on the number of uninfected RBCs. It shows that the number of uRBCs increases with immune cells' lifespan. Hence, using Figure 10 we argue that introducing a long-term immunity may significantly reduce the infection.

With reference to basic reproduction number, $\mathscr{R}_{0}$; as expressed in equation (9) it is noted that $\mathscr{R}_{0}$ can be made less than or equal to unity by: decreasing the infection rate, $\beta_{2}$ of uRBCs by merozoites; increasing death rates of iRBCs, blood-schizonts and merozoites; increasing the rates at which iRBCs, blood-schizonts and merozoites are cleared by immune cells; and increasing the rate at which immune cells suppress the production of merozoites from blood-schizonts. Therefore, any biological means that can be implemented to facilitate these may have impact on development of control strategies.

\section{Conclusion}

In this work, we have formulated and analyzed a mathematical model for the in-human host and in-human dynamics of malaria parasite with immune responses. In this model, we include the effect of immune responses to block invasion of sporozoites and merozoites on hepatic liver cells and red blood cells respectively. The effect of immune responses to inhibit the production of merozoites from both liver and blood cells was included. Additionally, the model incudes terms 


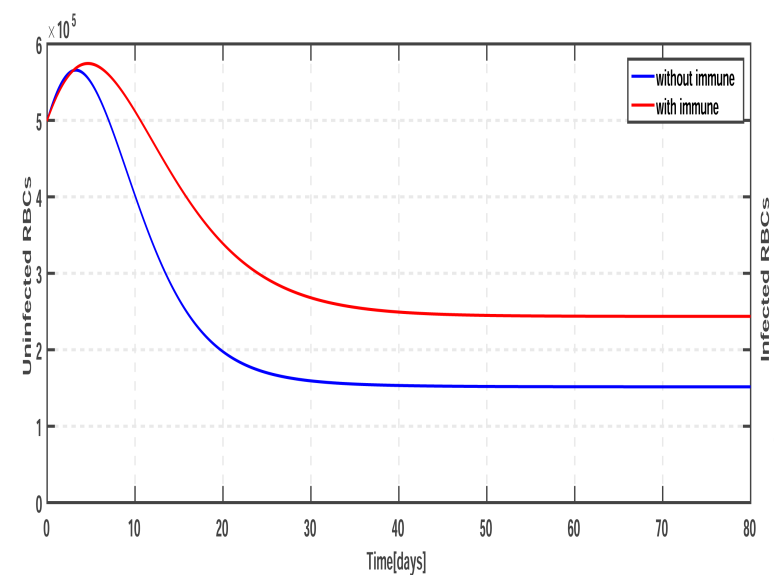

(a) Uninfected RBCs

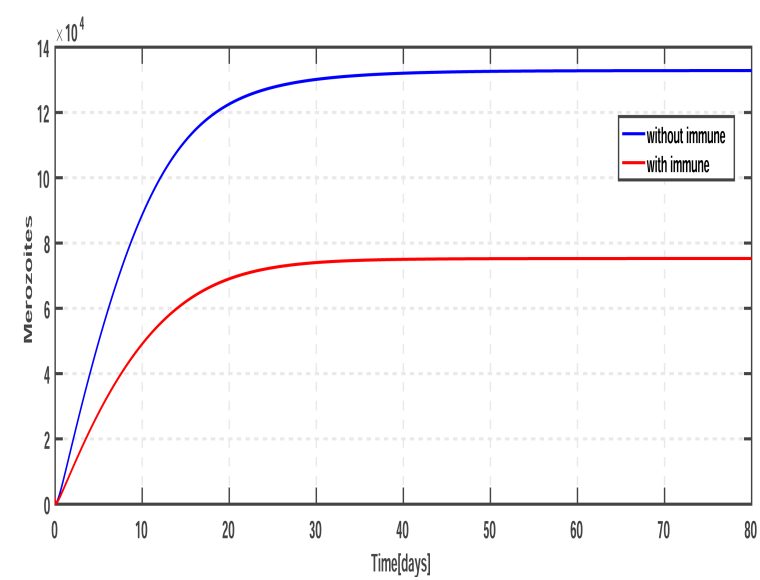

(c) Merozoites

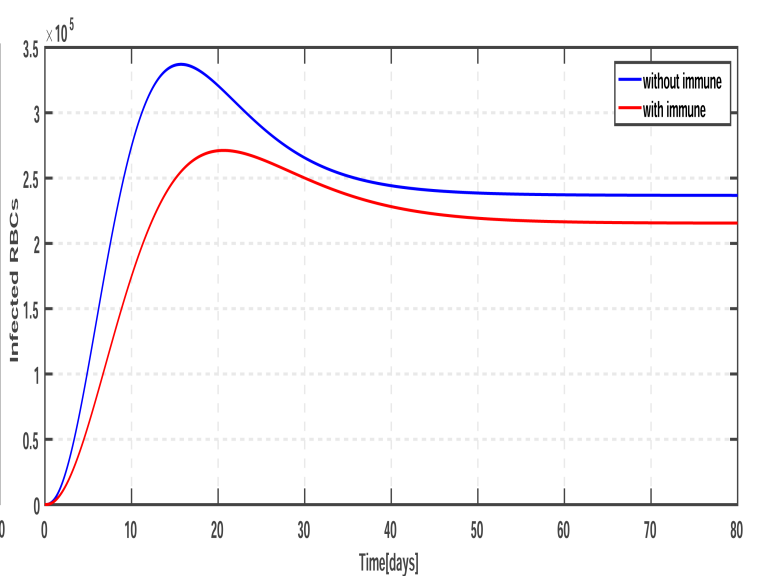

(b) Infected RBCs

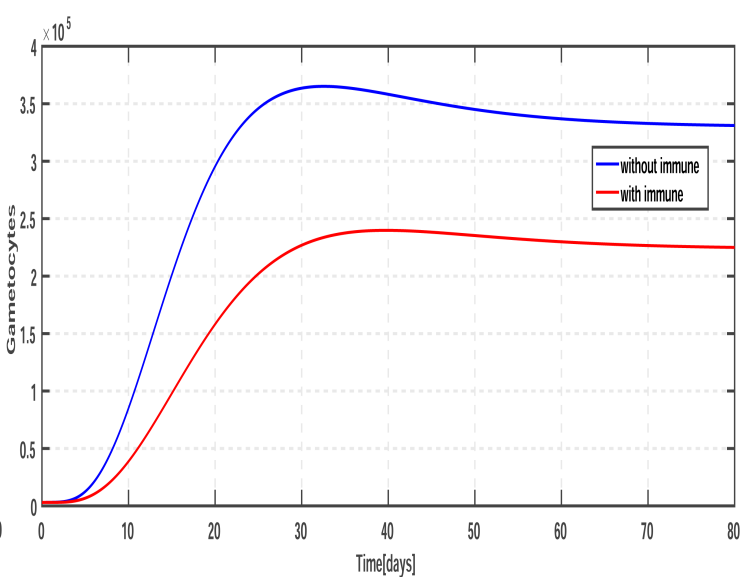

(d) Gametocytes

Fig. 7: Effects of immune responses on uninfected RBCs, infected RBCs, merozoites and gametocytes.

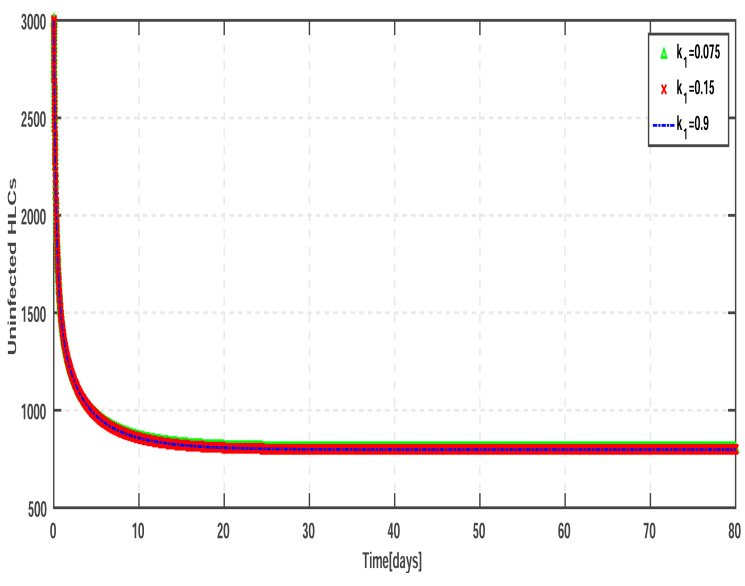

(a) Unifected HLCs

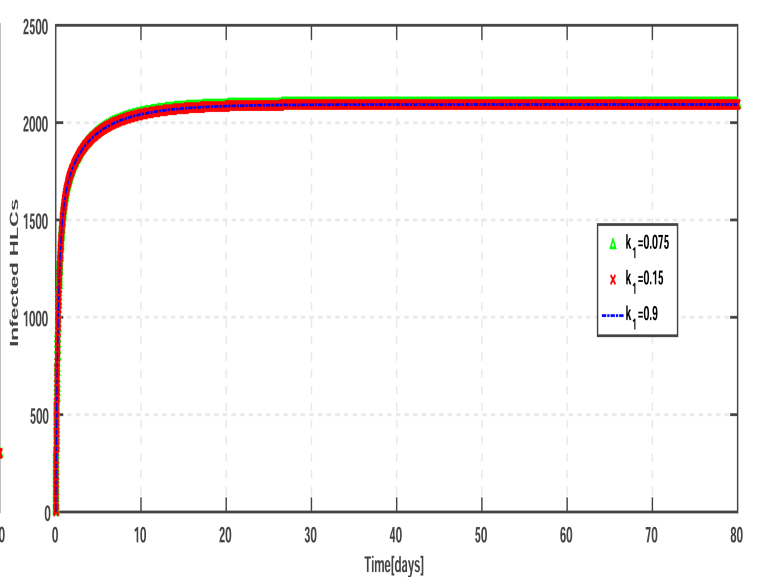

(b) Infected HLCs

Fig. 8: Graphs of liver-stage dynamics showing effect of immune responses. 


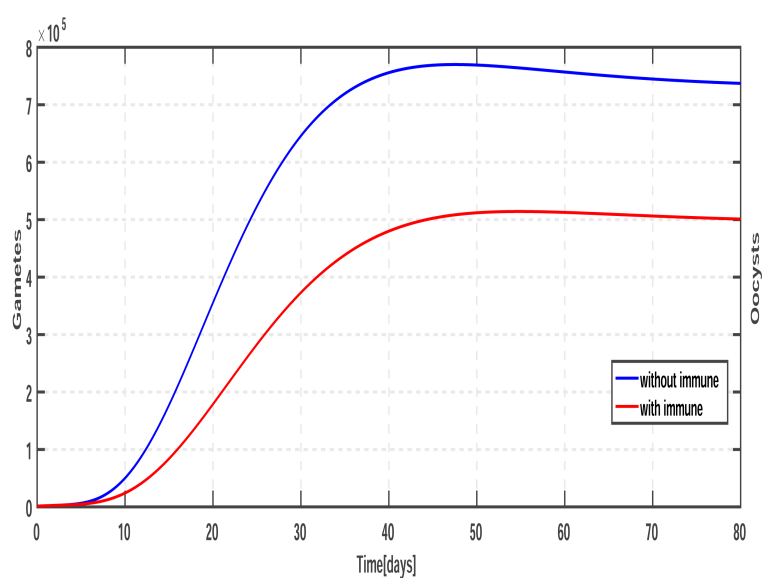

(a) Gametes

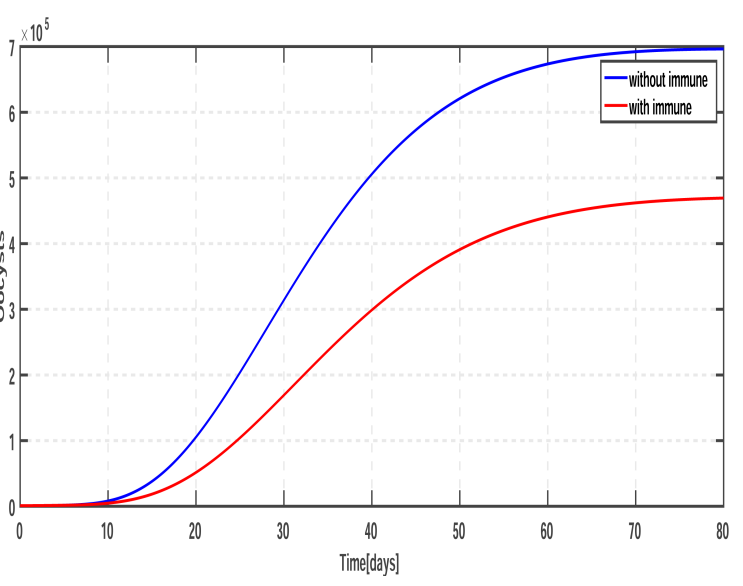

(b) Oocysts

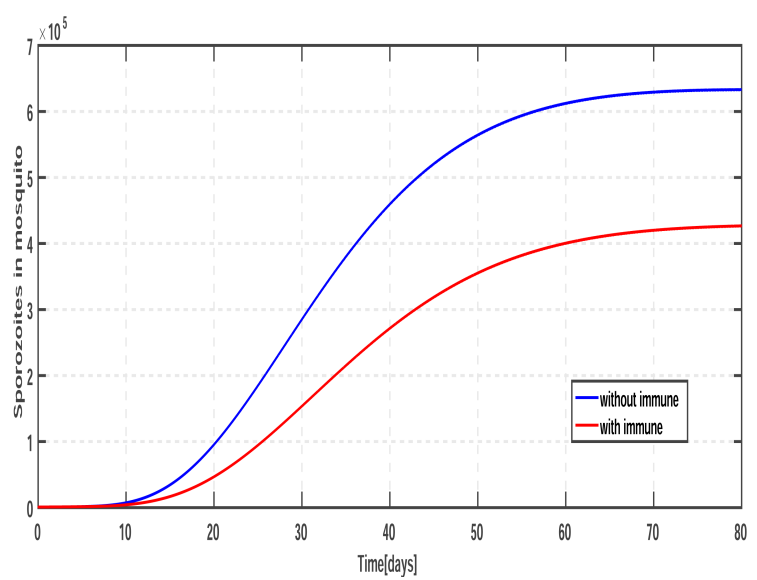

(c) Sporozoites in mosquito

Fig. 9: Effects of antibodies taken up by mosquito during the blood meal on gametes, oocysts and sporozoites.

for influence of immune responses on clearance of both parasites and infected cells. Finally, we include the term for effect of antibodies on gametocytes picked-up during the blood meal. In all cases immune response are described using the nonlinear-bounded Michaelis-Menten-Monod function.

A positive invariant region, where the model is epidemiologically (variables biological interpretation meaningful) and mathematically (always a unique bounded solution exists) well-posed was established. Using the next generation method, basic reproduction number $\mathscr{R}_{0}$, of the model was computed. Also, existence and stability of two non-negative equilibrium points: malaria free equilibrium (MFE) and malaria infection equlibrium (MIE) were established. Furthermore, we proved that MFE is locally asymptotically stable if $\mathscr{R}_{0}<1$ and globally asymptotically stable (GAS) if $\mathscr{R}_{0} \leq 1$. In addition to that, we noted that the impact of immune cells to suppress production of merozoites is higher than that of antibodies to block invasion of sporozoites and merozoites into liver and blood cells respectively. This is because none of $k^{\prime} s$, efficiency of antibodies to inhibit invasion appeared into the expression of $\mathscr{R}_{0}$, while one of the $c^{\prime} s\left(c_{2}\right)$, efficiency of immune cells to suppress production of merozoites does appear.

Numerical simulations prove the existence of MIE, which is GAS irrespective of the initial values do state variables have. Moreover, in comparison with the results of [19], our results revealed that including immunity has significant 


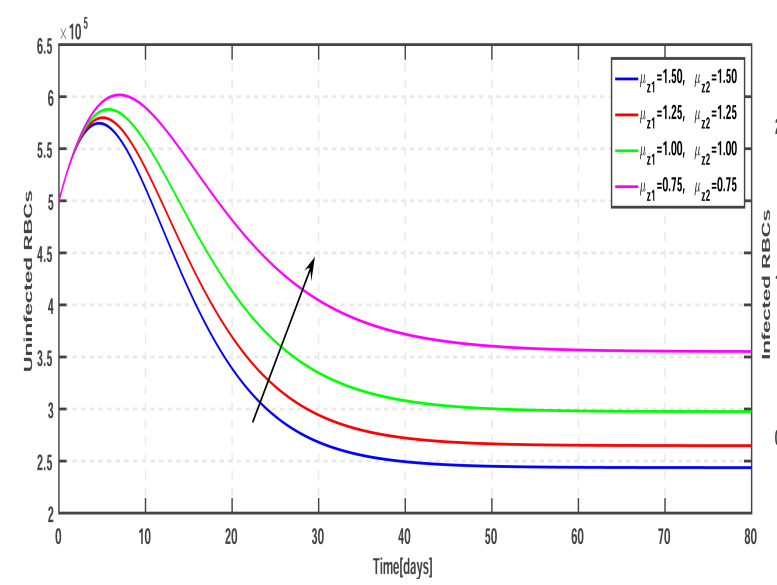

(a) Uninfected RBCs

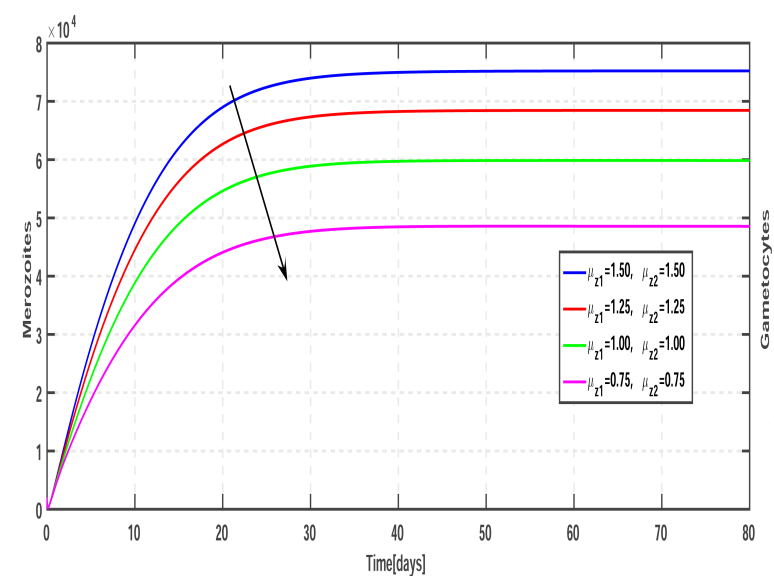

(c) Merozoites

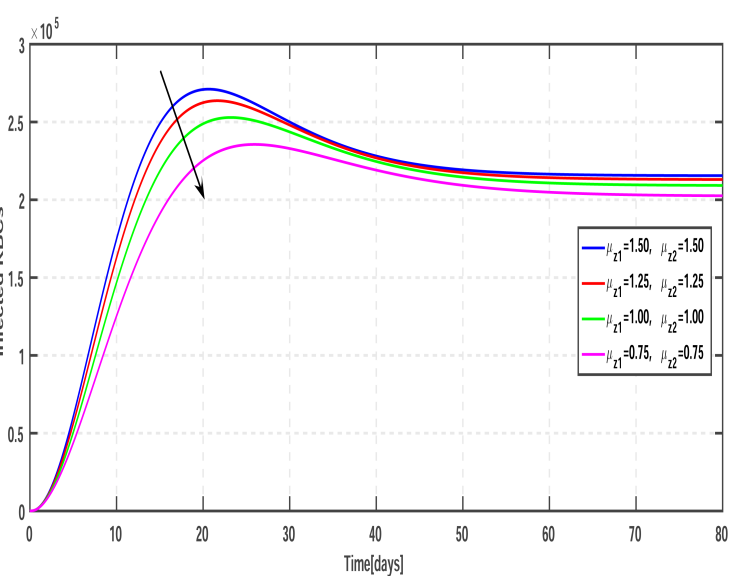

(b) Infected RBCs

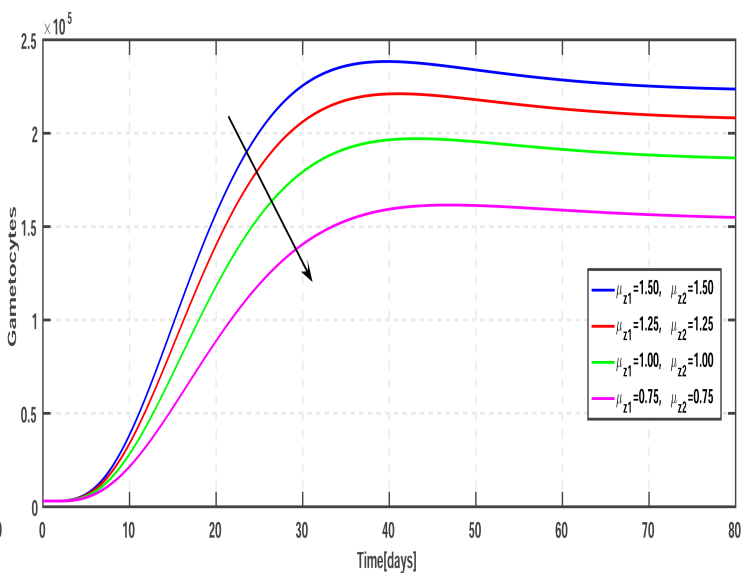

(d) Gametocytes

Fig. 10: Effects of life span of immune responses on uRBCs, iRBCs, merozoites and gametocytes. Arrows are in direction of increasing life span $\left(1 / \mu_{z_{2}}\right)$ of immune responses against blood stage infection.

influence on lowering infection at blood and mosquito stages. An increase on number of uninfected cells, and a decrease on number of infected cells and free parasite were noted. Also, antibodies picked-up by mosquito seems to have an effect on reducing the number of parasites within mosquito.

Based on the above results, this study suggests that the a combined drug therapy should be boosted so as to improve their ability to suppress parasite's production in bloodstream. Also, causing lysis to gametocytes is of great importance in preventing parasite' development in mosquito as it may lead us to a population of non-infectious mosquito. Hence, reduce mosquito-human infection.

Our work is an example on how mathematical models can be used to get insight of complex systems like malaria life cycle. Therefore, it provides basis for mathematical models to study the in-host and in-mosquito dynamics malaria and immunity. In future work we propose the extension this model by incorporating the effect of antimalaria drugs. 


\section{Competing interests}

The authors declare that they have no competing interests.

\section{Authors' contributions}

All authors have contributed to all parts of the article. All authors read and approved the final manuscript.

\section{References}

[1] Folashade B Agusto, Nizar Marcus, and Kazeem O Okosun. Application of optimal control to the epidemiology of malaria. Electronic Journal of Differential Equations, 2012(81):1-22, 2012.

[2] RM Anderson, RM May, and S Gupta. Non-linear phenomena in hostparasite interactions. Parasitology, 99(S1):S59-S79, 1989.

[3] K Artavanis-Tsakonas, JE Tongren, and EM Riley. The war between the malaria parasite and the immune system: immunity, immunoregulation and immunopathology. Clinical \& Experimental Immunology, 133(2):145-152, 2003.

[4] DJ Austin, NJ White, and RM Anderson. The dynamics of drug action on the within-host population growth of infectious agents: melding pharmacokinetics with pathogen population dynamics. Journal of theoretical biology, 194(3):313-339, 1998.

[5] Carlos Castillo-Chávez, Zhilan Feng, and Wenzhang Huang. On the computation of $R_{0}$ and its role in global stability. In Carlos Castillo-Chávez, Sally Blower, Pauline Van den Driessche, Denise Kirschner, and Abdul-Aziz Yakubu, editors, Mathematical Approaches for Emerging and Reemerging Infectious Diseases: An Introduction, pages 229-250. Springer, 2002.

[6] C Chiyaka, W Garira, and S Dube. Modelling immune response and drug therapy in human malaria infection. Computational and Mathematical Methods in Medicine, 9(2):143-163, 2008.

[7] Giampietro Corradin and Jelena Levitskaya. Priming of CD8 ${ }^{+} \mathrm{T}$ cell responses to liver stage malaria parasite antigens. Frontiers in Immunology, 5(527):1-6, 2014.

[8] Dari F Da, Thomas S Churcher, Rakiswendé S Yerbanga, Bienvenue Yaméogo, Ibrahim Sangaré, Jean Bosco Ouedraogo, Robert E Sinden, Andrew M Blagborough, and Anna Cohuet. Experimental study of the relationship between plasmodium gametocyte density and infection success in mosquitoes; implications for the evaluation of malaria transmission-reducing interventions. Experimental parasitology, 149:74-83, 2015.

[9] Hans H Diebner, Martin Eichner, Louis Molineaux, William E Collins, Geoffrey M Jeffery, and Klaus Dietz. Modelling the transition of asexual blood stages of plasmodium falciparum to gametocytes. Journal of Theoretical Biology, 202(2):113-127, 2000.

[10] S Dube, Winston Garira, and Christinah Chiyaka. Using mathematics to understand malaria infection during erythrocytic stages. Zimbabwe Journal of Science and Technology, 5:1-11, 2010.

[11] Yves Dumont, Frédéric Chiroleu, and Caroline Domerg. On a temporal model for the chikungunya disease: Modeling, theory and numerics. Mathematical biosciences, 213(1):80-91, 2008.

[12] Michael F Good. Towards a blood-stage vaccine for malaria: Are we following all the leads? Nature Reviews Immunology, 1(2):117-125, 2001.

[13] Jean Claude Kamgang and Gauthier Sallet. Computation of threshold conditions for epidemiological models and global stability of the disease-free equilibrium (dfe). Mathematical biosciences, 213(1):1-12, 2008.

[14] Sam M. Kinyanjui. The immunology of malaria. In Omolade O. Okwa, editor, Malaria parasites, chapter 10, pages 175-201. InTech, 2012.

[15] Jean Langhorne, Francis M Ndungu, Anne-Marit Sponaas, and Kevin Marsh. Immunity to malaria: more questions than answers. Nature immunology, 9(7):725-732, 2008.

[16] Yilong Li, Shigui Ruan, and Dongmei Xiao. The within-host dynamics of malaria infection with immune response. Mathematical Biosciences and Engineering, 8(4):999-1018, 2011.

[17] Saul C Mpeshe, Livingstone S Luboobi, and Yaw Nkansah-Gyekye. Stability analysis of the rift valley fever dynamical model. Journal of Mathematical and Computational Science, 4(4):740-762, 2014. 
[18] Kenrad E Nelson and Carolyn Masters Williams. Infectious disease epidemiology: theory and practice. Jones \& Bartlett Publishers, Burlington, USA, 2014.

[19] Mohamed A Selemani, Livingstone S Luboobi, and Yaw Nkansah-Gyekye. On stability of the in-human host and in-mosquito of malaria parasites. Asian Journal of Mathematics and Applications, Vol. 2016:article ID ama0353, 23 pages, 2016.

[20] J Tumwiine, JYT Mugisha, and Livingstone S Luboobi. On global stability of the intra-host dynamics of malaria and the immune system. Journal of Mathematical Analysis and Applications, 341(2):855-869, 2008.

[21] Pauline Van den Driessche and James Watmough. Reproduction numbers and sub-threshold endemic equilibria for compartmental models of disease transmission. Mathematical biosciences, 180(1):29-48, 2002. 